\title{
A spatiotemporal complexity architecture of human brain activity
}

Stephan Krohn 1,2, ${ }^{*}$, Nina von Schwanenflug ${ }^{1,2, \#, ~ L e o n h a r d ~ W a s c h k e ~} 3,4$,\#, Amy Romanello1,2, Martin Gell2,5,6, Douglas D. Garrett ${ }^{3,4}$, Carsten Finke 1,2,

1Department of Neurology, Charité-Universitätsmedizin Berlin, Berlin, Germany, ${ }^{2}$ Berlin School of Mind and Brain, Humboldt-Universität zu Berlin, Berlin, Germany ${ }^{3}$ Center for Lifespan Psychology, Max Planck Institute for Human Development, Berlin, Germany ${ }^{4}$ Max Planck UCL Centre for Computational Psychiatry and Ageing Research, Berlin, Germany

5 Institute of Neuroscience and Medicine (INM-7), Heinrich Heine University Düsseldorf, Düsseldorf, Germany

${ }^{6}$ Department of Psychiatry, Psychotherapy and Psychosomatic Medicine, RWTH Aachen University, Aachen, Germany

\#equal contribution, alphabetical order

${ }^{*}$ Corresponding authors:

Stephan Krohn

Carsten Finke

Department of Neurology

Charité - Universitätsmedizin Berlin

Charitéplatz 1

10117 Berlin

Germany

stephan.krohn@charite.de

carsten.finke@charite.de

Keywords: brain activity, functional magnetic resonance imaging, human connectome, functional networks, neural variability, signal complexity 


\begin{abstract}
The human brain operates in large-scale functional networks, collectively subsumed as the functional connectome ${ }^{1-13}$. Recent work has begun to unravel the organization of the connectome, including the temporal dynamics of brain states ${ }^{14-20}$, the trade-off between segregation and integration ${ }^{9,15,21-23}$, and a functional hierarchy from lower-order unimodal to higher-order transmodal processing systems ${ }^{24-27}$. However, it remains unknown how these network properties are embedded in the brain and if they emerge from a common neural foundation.

Here we apply time-resolved estimation of brain signal complexity to uncover a unifying principle of brain organization, linking the connectome to neural variability ${ }^{6,28-31}$. Using functional magnetic resonance imaging (fMRI), we show that neural activity is marked by spontaneous "complexity drops" that reflect episodes of increased pattern regularity in the brain, and that functional connections among brain regions are an expression of their simultaneous engagement in such episodes. Moreover, these complexity drops ubiquitously propagate along cortical hierarchies, suggesting that the brain intrinsically reiterates its own functional architecture. Globally, neural activity clusters into temporal complexity states that dynamically shape the coupling strength and configuration of the connectome, implementing a continuous re-negotiation between cost-efficient segregation and communicationenhancing integration $9,15,21,23$. Furthermore, complexity states resolve the recently discovered association between anatomical and functional network hierarchies comprehensively ${ }^{25-27,32}$. Finally, brain signal complexity is highly sensitive to age and reflects inter-individual differences in cognition and motor function. In sum, we identify a spatiotemporal complexity architecture of neural activity - a functional "complexome" that gives rise to the network organization of the human brain.
\end{abstract}




\section{Main}

The human brain operates in large-scale functional networks thought to underlie cognition and behavior ${ }^{1-13}$. Collectively subsumed as the functional human connectome, these networks arise from the functional connectivity (FC) among brain regions, estimated as the correlation structure of whole-brain activity in $\mathrm{fMRI}^{1,2,28,33,34}$. The organization of these connectivity networks is increasingly well understood, including the temporal dynamics ${ }^{14-20}$, network economy ${ }^{21-23}$, and functional hierarchy ${ }^{24-27}$ within the connectome. However, it remains a fundamental challenge in neuroscience to understand how these spatially distributed properties are embedded in the local activity of individual brain regions ${ }^{8,29}$. Recent work suggests that functional connections between regions arise from only a few critical episodes in the blood oxygen level-dependent (BOLD) brain signals ${ }^{17,35,36}$, yet the nature of such critical instants remains unclear.

Here we approach these challenges through the variability of neural activity, which has been hypothesized to enable the formation of the connectome $e^{5,6,8,29,37}$, and which is increasingly recognized to carry important functional links to age and behavior ${ }^{30,31,38-40}$. Using timeresolved complexity estimation of resting-state BOLD signals, we uncover the universal phenomenon of "complexity drops" that represent spontaneous episodes of increased pattern regularity in the brain. Identifying such critical episodes on the level of individual brain regions allows for the desired link between the neural activity of a particular region (i.e., the nodes of the connectome) and the inter-regional network properties that emerge from such activity (i.e., the edges of the connectome). Accordingly, we first tested the link between complexity drops and functional connections and found that FC among brain regions is an expression of their simultaneous engagement in such episodes, and that the brain's network structure reflects this inter-regional drop coincidence.

Furthermore, a recent line of research points to the infra-slow propagation of BOLD activity patterns throughout the brain ${ }^{41-46}$, offering a potential mechanism for trans-network communication ${ }^{47}$. As complexity drops capture precisely such signal patterns, we next turned to the temporal evolution of these episodes and confirmed a lawful propagation of complexity drops across the brain. Intriguingly, these propagations not only adhered to the brain's network architecture, but also intrinsically followed the recently reported unimodalto-transmodal functional hierarchy ${ }^{20,24-26}$, suggesting that the brain spontaneously and repeatedly reiterates its own functional architecture. 
Moreover, mounting evidence suggests that the connectome is temporally dynamic, marked by a continuous alternation between functional network states ${ }^{14-20}$. Given the link between complexity drops and FC, we hypothesized that such states of the network may be rooted in underlying states of neural activity. Supporting this idea, we found that the brain operates in temporal complexity states, and that these states result in gradual and momentary changes in the coupling strength of the connectome. Apart from connectivity alone, however, we expected that the topology of the connectome might be similarly adaptive, given that the brain is thought to balance cost-efficient segregated network configurations with communication-enhancing integrated configurations ${ }^{9,21,23}$. Supporting the notion of such a cost-efficiency trade-off ${ }^{9,21}$, we found that the default state of neural activity is a low-cost state marked by few complexity drops and high segregation, and that this state is occasionally interrupted by high-efficiency brain states featuring many drops and high integration within the connectome.

Furthermore, recent work on spatial network embedding has revealed a functional hierarchy within the connectome, characterized by a principal gradient between lower-order unimodal and higher-order transmodal processing systems ${ }^{24-26}$. Given its link to complexity drop propagation, we next tested the spatial relationship between this gradient and complexity states and corroborated a strong convergence of their topologies. In addition, this gradient measure of functional hierarchy has been linked to proxies of anatomical hierarchy such as cortical myeloarchitecture $27,32,48,49$. Since complexity state topology represents both a local brain characteristic (like myelination) and a functional measure (like gradient loadings), we hypothesized that complexity states may bridge such a structure-function relationship within the network ${ }^{50}$. Indeed, accounting for complexity state topology completely resolved the gradient-myelin association, but not vice versa, suggesting that complexity state dynamics may underlie the link between microstructure and connectivity.

Lastly, we turn to the behavioral implications of our findings, given that the variability of neural activity is increasingly recognized to be functionally relevant ${ }^{29-31,38-40,51-53}$. Even over the narrow range of early adulthood, complexity dynamics were highly sensitive to age and reflected inter-individual differences in cognition and motor performance, suggesting that an increased capacity for complexity drops represents a beneficial functional profile of the brain.

Below, we address these analyses in turn, combining multimodal imaging data from the Human Connectome Project (HCP) $)^{54,55}$ with analytical approaches including graph theory, structure detection, resampling methods and multivariate techniques. 


\section{Resting-state complexity dynamics}

Complexity dynamics were estimated through time-resolved symbolic encoding ${ }^{56,57}$ on the BOLD signals, yielding a complexity timeseries for each region of interest (Fig.1a). This approach leverages both pattern diversity and amplitude information within the signal (Extended Data Fig.1-2) to reveal a universal complexity structure of brain activity: Over about $80 \%$ of acquisition time, the brain exhibits widespread high-complexity signals, approaching the upper limit of expected values given the underlying signal characteristics (Extended Data Fig.1+3). Intermittently, however, this high-complexity activity is interrupted by spontaneous "complexity drops" that correspond to transient episodes of increased pattern regularity in the signals (Fig.1a, inset). These complexity drops appear in clusters of brain regions and over well-delineated periods in time, and the number of brain regions engaging in them closely reflects the brain's overall signal complexity at any given point in time. Notably, these complexity dynamics were ubiquitously present in all analyzed recordings (684 scans from 343 participants; see online repository: https://osf.io/mr8f7) and were highly robust against different windowing parameters and parcellation approaches (Extended Data Fig.4-5). Spatial analysis revealed continuous high-complexity activity in medial temporal, anterior cingulate and subcortical regions -most markedly thalamic areas $^{58}$-, while complexity drops predominantly occurred in the cortex, with a focus on pericentral regions (Fig.1b). On the level of individual participants, the affinity for complexity drops was strongly linked to overall signal complexity $(r=-0.9, p=1.6 e-125)$ and showed significant constraints by established resting-state networks ${ }^{7}\left(R S N ; X^{2}(7)=154, p=6.1 e-30\right)$ as well as gradual decreases with age $\left(X^{2}(2)=17.1, p=1.9 e-4\right)$, even over the narrow age range of early adulthood (Fig.1b).

In line with the idea that FC emerges from only a few critical episodes in the BOLD signals ${ }^{17,35,36}$, regional complexity dynamics were intrinsically linked to inter-regional connectivity. Analyzing the occurrence of complexity drops revealed that the FC between any two regions is strongly driven by the degree to which they engage in complexity drops simultaneously (Fig.1c). While this "drop coincidence" explained a large part of the variance in $F C$ overall $\left(F(1,30133)=4.3 e 4, p \approx 0, R^{2} a d j=0.59\right)$, it was also significantly higher for connections within, rather than across canonical $R S N s(W=3.6 e 7, p=9.3 e-161)$, suggesting that the brain's network structure is a reflection of inter-regional differences in simultaneous complexity drops.

Further supporting this link to network differentiation, complexity timeseries exhibited a varying degree of interhemispheric symmetry (Fig.1d). Complexity symmetry was 
systematically constrained by RSNs $\left(X^{2}(7)=169, p=4.6 e-33\right)$ and intrinsically followed the recently uncovered functional hierarchy between unimodal and transmodal areas ${ }^{24-27}$, suggesting that the cross-hemispheric coupling of brain activity patterns is strongest in primary networks and becomes gradually more diverse in higher-order systems.
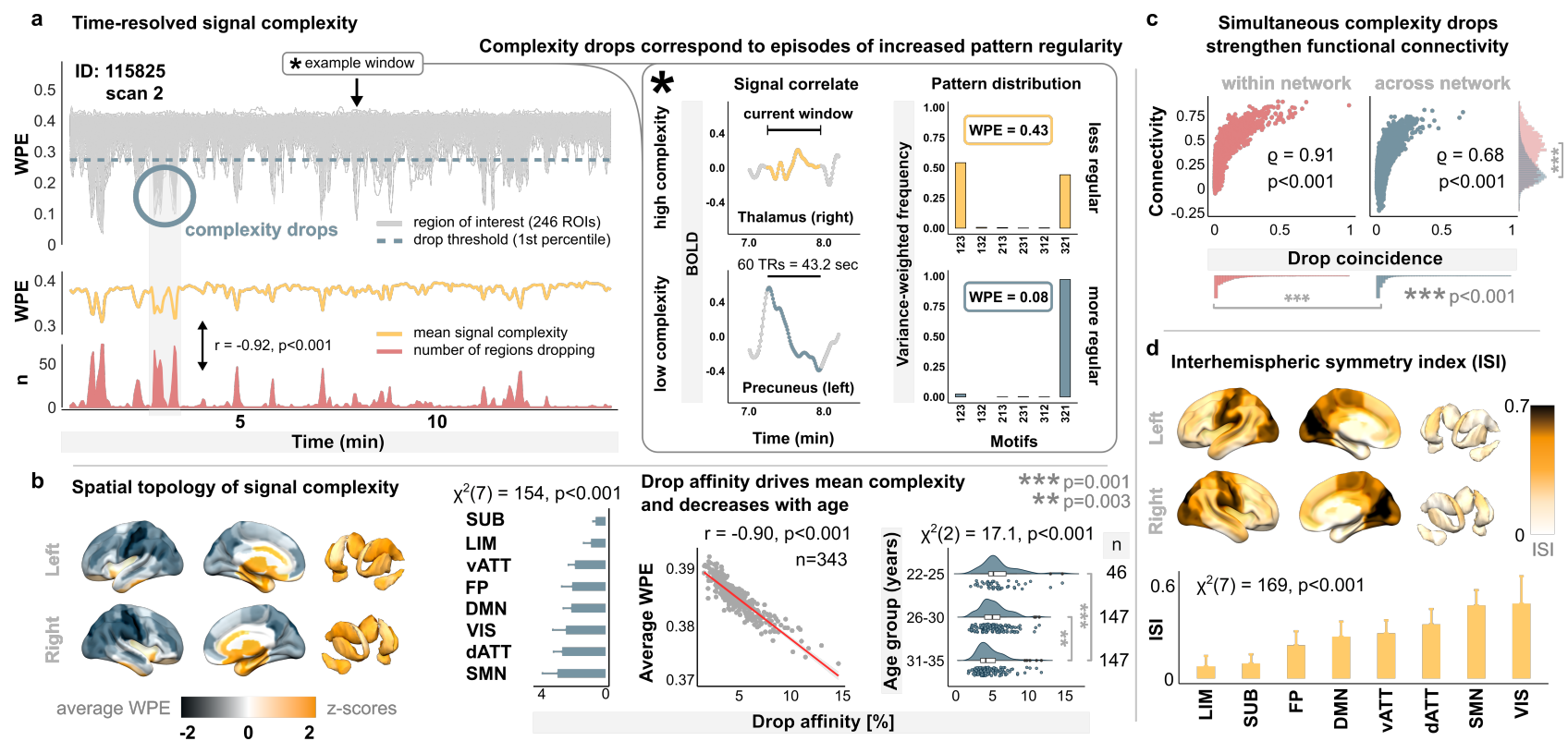

Fig.1 | Time-resolved signal complexity of human brain activity. a, Weighted permutation entropy (WPE) timeseries for each region of interest (ROI) from an exemplary resting-state recording (Brainnetome parcellation ${ }^{59}$; windows length $60 \mathrm{TRs}, 95 \%$ overlap). The inset displays the BOLD correlates of exemplary high- and low-complexity signals. b. Spatial distribution of average signal complexity. Drop affinity over RSNs, link to grand-average signal complexity, and age effect. c, Drop coincidence and inter-regional FC, within and across canonical RSNs ${ }^{7}$. d, Spatial topology and network-wise distribution of interhemispheric symmetry. Network abbreviations: dATT: dorsal attention; DMN: default mode; FP: frontoparietal; LIM: limbic; SMN: somatomotor; SUB: subcortical; VATT: ventral attention; VIS: visual.

\section{Complexity drops spread across the brain}

In view of accumulating evidence for the propagation of BOLD activity patterns throughout the brain ${ }^{41-47}$, we next probed such a pattern propagation in the temporal evolution of complexity drops. In line with this idea, complexity drops consistently occurred in dynamic cascades that start with a few initializing regions, gradually spread across the brain, and finally fade back to a few regions again, such that brain-wide drop engagement exhibits an inverted U-shape (Fig.2a, online video: https://osf.io/mr8f7). To formalize the spread of complexity drops across the brain, we developed a graph theoretical framework, where each individual propagation is described by a directed graph. These propagation graphs contain node layers that represent consecutive BOLD windows from cascade initialization ("source 
layer") to maximum drop engagement ("peak layer"), and where the direction of edges codes for progression in time (Fig.2b), enabling the estimation of region-to-region transition probabilities within a propagation.

Drop cascades were characterized by a strong positive relationship between cascade duration and the number of regions engaging in them $\left(\rho=0.79, p_{F D R}=2.9 e-73\right)$, and the number of cascades an individual presented was strongly related to their source diversity, defined as the proportion of brain regions ever initializing a cascade $\left(\rho=0.96, p_{F D R}=2 e-182\right.$; Fig.2c). Notably, we observed significant age-related decreases in the number of cascades $\left(X^{2}(2)=13.9, p=9.5 e-4\right)$ and in source diversity $\left(X^{2}(2)=16.3, p=2.8 e-4\right)$, but not in the number of regions dropping $\left(X^{2}(2)=4.5, p=0.11\right)$ or cascade duration $\left(X^{2}(2)=2.6, p=0.27\right)$, suggesting that individual propagations occur in a canonical fashion, although the predisposition to engage in them decreases with age (Fig.2c).

Spatial analysis of propagation graphs revealed that drop cascades originate predominantly in the cortex, with a focus on lower-order RSNs (Fig.2d). Furthermore, graph construction from the transition probabilities of individual spreads uncovered a highly structured propagation network (Fig.2e). Centrality analysis of this network revealed that the propagation of complexity drops follows an intrinsic hierarchy (Fig.2f), where the most influential nodes correspond to primary RSNs, and nodes of higher-order systems become gradually less central to the propagation.

Further supporting this propagation hierarchy, geodesic distances in the network showed that complexity drops spread along a unimodal-to-transmodal functional gradient (Fig.2g; Monte Carlo simulation of cluster groups: $\left.x_{\text {cluster }}=0.34, p=0.006\right)$. Furthermore, modelling geodesics as a function of probabilistic streamline connectivity ${ }^{60}$ and number of structural links $\left(R_{a d j}{ }^{2}=0.66, F(2,18)=20.9, p=2 e-5\right)$ significantly improved explanatory power $\left(\Delta R^{2}=0.3\right.$, $\eta^{2}$ partial $\left.=0.5, F(1,18)=17.8, p=5.1 e-4\right)$ compared to when only the number of links were considered $\left(\mathrm{R}_{\mathrm{adj}}{ }^{2}=0.37, \mathrm{~F}(1,19)=12.7, \mathrm{p}=0.002\right)$, suggesting that the propagation of complexity drops is further constrained by structural connectivity.

These findings strongly converge with the recently uncovered hierarchy within the connectome ${ }^{24-27}$, characterized by a principal gradient between lower-order unimodal and higher-order transmodal processing systems. Additionally, the propagation of complexity drops aligns well with the recently reported activity pulses in disused motor circuits ${ }^{45}$, which likewise occurred spontaneously and were propagated through the motor network. 
Our findings suggest that these pulses represent an adaptive enhancement of a preexisting phenomenon, the dynamic propagation of regularity throughout the brain. Given that these propagations intrinsically followed the brain's functional hierarchy, such episodes may not be limited to impairment-induced plasticity ${ }^{45}$ but rather represent a more general mechanism by which the brain repeatedly and adaptively reiterates its own functional architecture.

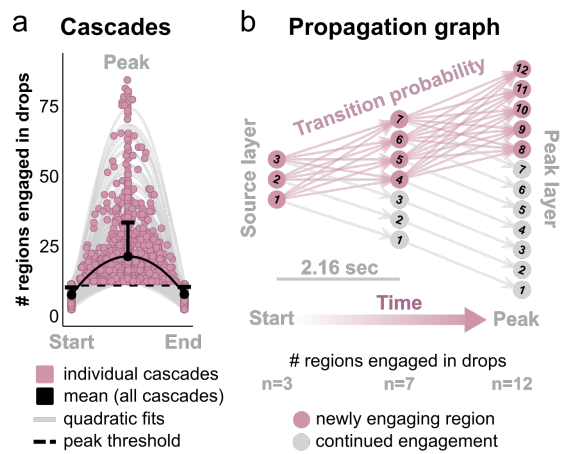

d Cascade origination

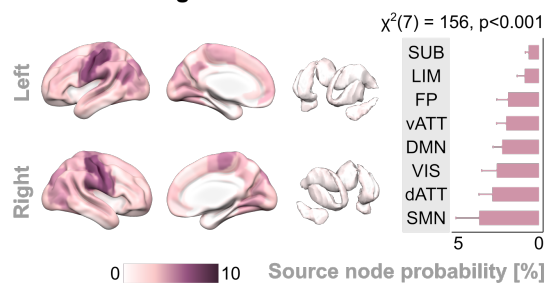

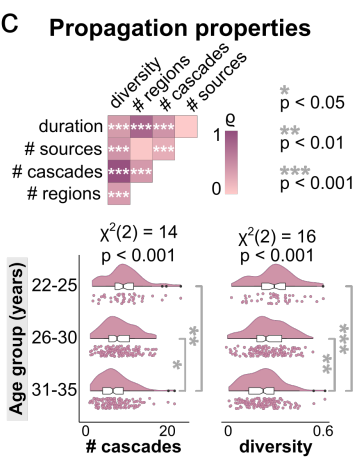

e Propagation network

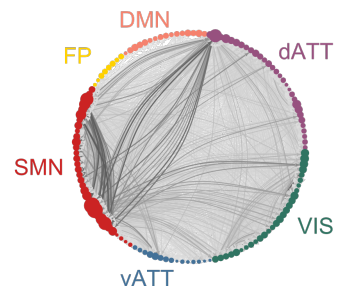

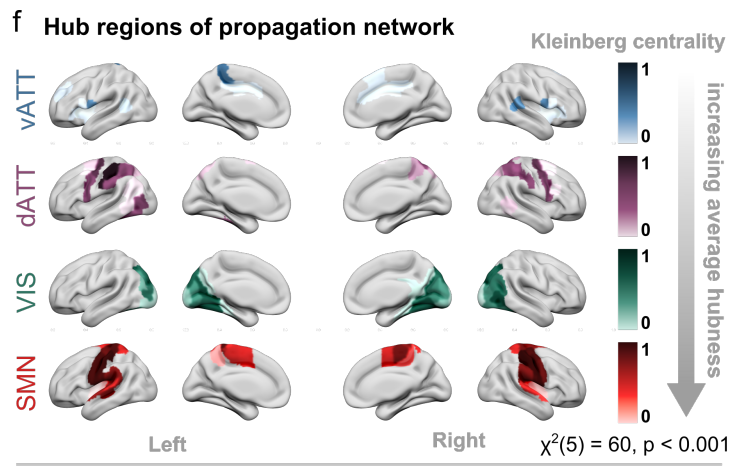

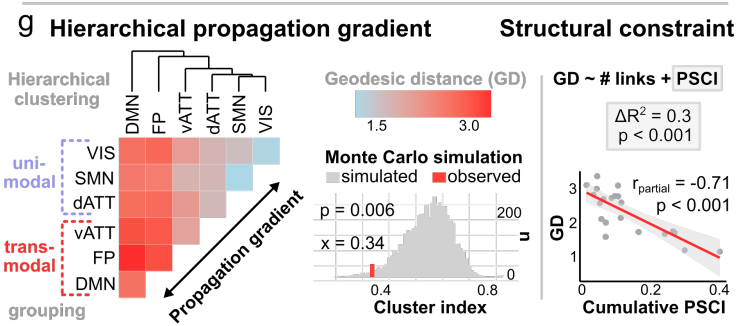

Fig. 2 | Complexity drops spread across the brain. a, Drop cascades. Points represent a random sample of 500 out of 5345 total cascades in the study population. Engagement is thresholded to at least 10 regions dropping simultaneously at peak. b. Formalization of individual spreads as directed propagation graphs. Window-to-window temporal resolution corresponds to 2.16 seconds (3 TRs). c, Propagation properties and age effects (pairwise tests FDR-corrected). d, Spatial topology of cascade origination. e, Propagation network, thresholded to the $95^{\text {th }}$ percentile of strongest connections (edge thickness: transition probability; node diameter: source probability). f, Hub topology of the propagation network, given by Kleinberg's eigenvector centrality. FP and DMN are omitted because of near-zero entries only. g. Average node-to-node geodesic distances in the propagation network. Monte Carlo simulation testing the significance of unimodal vs. transmodal network clusters. Association between geodesic distances and the probabilistic streamline connectivity index (PSCl).

\section{The brain operates in complexity states}

Next, we tested the idea that the temporal dynamics of functional network states ${ }^{14-20}$ may be rooted in underlying states of neural activity. Unsupervised clustering on the complexity timeseries supported this hypothesis, suggesting that the brain operates in distinct complexity states. In line with the inspection of individual timeseries (Fig.1a; online repository), participants spent most time in a default high-complexity state (Fig.3a), while the more infrequently visited states entailed gradually decreasing levels of complexity, 
yielding a strong discriminatory effect of complexity across states $\left(\eta^{2}=0.90,[0.89-0.91]\right)$. Notably, while complexity states are derived directly from the activity of individual brain regions, they resulted in pronounced effects on the coupling strength and configuration of the connectome, which are derived from the inter-regional correlation of activity. In consequence, the default state of neural activity was marked by low coupling strength of the network, while the lower-complexity states entailed gradual increases in connectivity.

The impact of complexity states furthermore extended to the configuration of the connectome ${ }^{21-23}$, yielding high network modularity and low efficiency in high-complexity brain states. As for connectivity, lower-complexity states resulted in gradual decreases in modularity and concordant increases in network efficiency. This impact of complexity states on the network also held at the temporal resolution of individual window-to-window transitions (Fig.1b). While all complexity states were similarly stable, switches between lowcomplexity and high-complexity brain states typically occurred through one of the intermediate complexity states, and these transitions were accompanied by fine-grained changes in the connectivity, modularity, and efficiency of the network.

These findings show that complexity states of neural activity shape the coupling strength and topology of the connectome in a moment-by-moment fashion. Notably, modularity and efficiency represent two complimentary network characteristics, where the former describes a segregated configuration thought to reduce biological cost and the latter implies an integrated configuration that enhances communication within the network ${ }^{9,21,22,61}$. The brain must balance both, however, yielding a cost-efficiency trade-off that is continuously renegotiated ${ }^{21}$. Our findings indicate that the brain implements such a trade-off with a default state of segregated neural activity that maintains cost-effectiveness, while the infrequent low-complexity states ensure recurrent phases of high coupling and functional integration.

Given these effects of complexity states on the network, we furthermore tested how much the space of possible complexity states (defined by clustering parameters, see Methods) was explored by individual participants (Fig.3c). To this end, we derived a measure of state exploration based on the difference between an individual's empirical state visits and a theoretical uniform distribution. This state dispersion index (SDI) showed good betweenscan reliability and significantly decreased with age $(X 2(2)=12.8, p=0.002)$, suggesting that older participants show increasingly rigid dynamics of neural activity, consistent with the age-related reductions in drop affinity (Fig.1b) and propagation diversity (Fig.2c). 
Notably, all state findings were highly robust against the number of expected complexity states (Extended Data Fig.6-7).

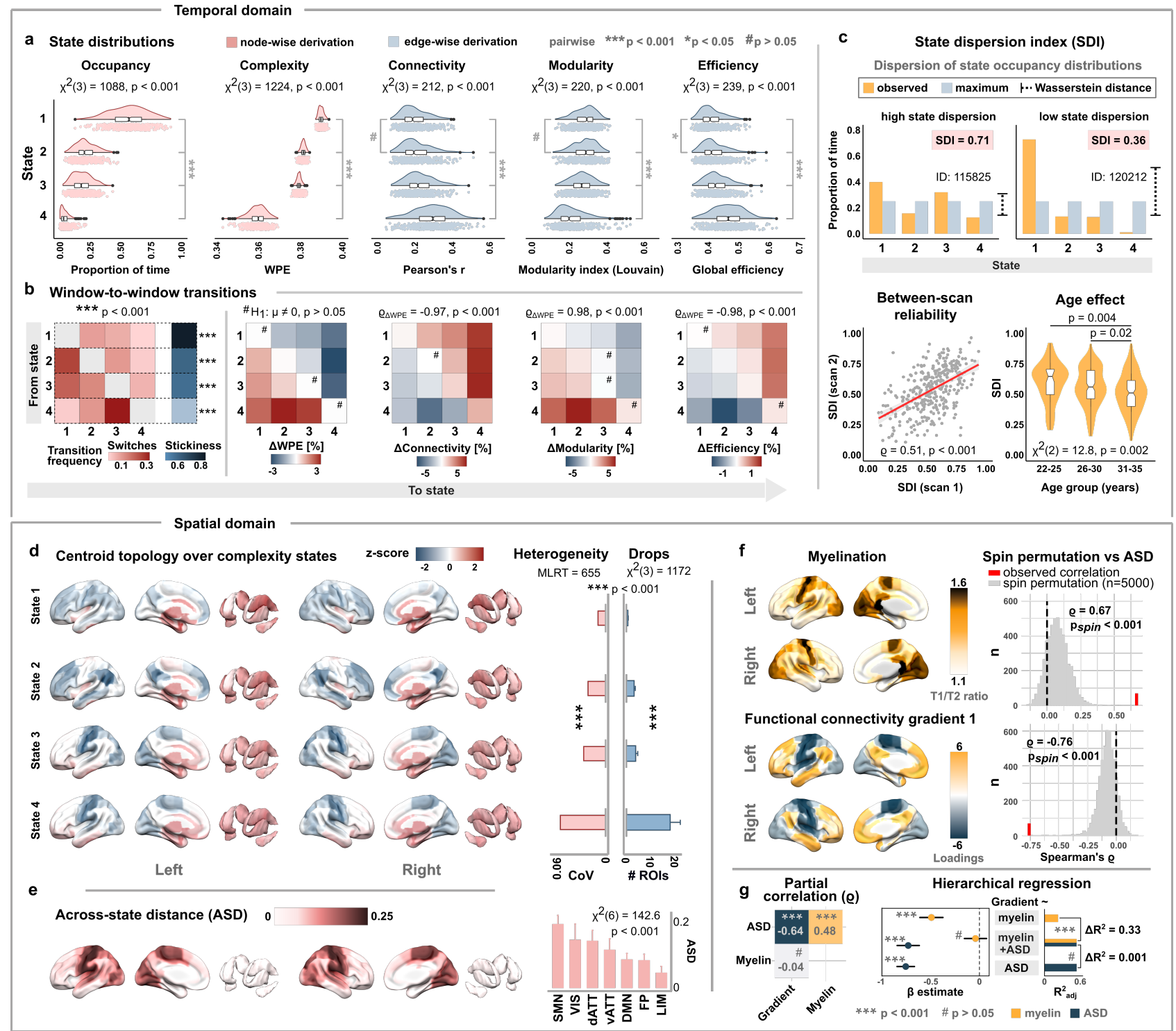

Fig.3 | The brain operates in complexity states. a, State-wise distributions of occupancy, signal complexity, FC strength, network modularity, and global efficiency $(n=343)$. b. Window-to-window state transition frequencies. State-dependent changes tested against deviation from zero (t-test, $\mathrm{p}_{\mathrm{FDR}}<0.05$ for all except those marked by \#). Association of network measures with $\triangle \mathrm{WPE}$ correspond to the correlation of the respective matrices. c, Illustration, reliability, and age effect of the state dispersion index (SDI). d, Spatial topology of centroid locations (within-state z-scores). Coefficient of variation (CoV) over regions and statewise complexity drops. MLRT, modified likelihood ratio test. e, Topology and network distribution of the across-state distance (ASD). $\mathbf{f}$, Average regional myelination and the unimodal-to-transmodal FC gradient. Correlation to ASD assessed by spin permutation tests. $\mathbf{g}$, Partial correlation between ASD, myelin, and the connectivity gradient. Hierarchical regression on the gradient loadings with myelin content and ASD as explanatory variables. All pairwise tests FDR-corrected. 


\section{Complexity states and network hierarchy}

Next, we tested the topology of complexity states against recent insights into the spatial embedding of the connectome ${ }^{25,26}$. Corroborating the distribution of average signal complexity (Fig.1b), subcortical areas persistently showed high-complexity activity, largely independent from the current global complexity state (Fig.3d). In contrast, cortical topologies varied distinctly with the number of regions engaged in complexity drops $\left(x^{2}(3)=1172\right.$, $p=9.4 e-254)$, inducing significant spatial heterogeneity across complexity states (MLRT=655, $\mathrm{p} \approx 0$ ).

To investigate which brain regions drive these differences, we derived a measure of acrossstate distance (ASD) as the cumulative centroid-to-centroid Euclidean distance for every region across 4-dimensional state space (Fig.3e). Consistent with the interhemispheric signal coupling (Fig.1) and the propagation gradient of complexity drops (Fig.2), the ASD intrinsically followed a unimodal-to-transmodal spatial gradient ${ }^{25,26}$, where regions that were most variable across complexity states represented the unimodal end of the hierarchy. To test this spatial convergence, we estimated both the connectivity gradient ${ }^{24,25,49}$ as well as cortical myelination ${ }^{48}$, a proxy of anatomical hierarchy that has been linked to these gradient loadings ${ }^{26,27,32,49}$, yielding a structure-function relationship within the connectome ${ }^{50}$.

Spin permutation testing ${ }^{49,62}$ uncovered a pronounced positive association of ASD topology with cortical myeloarchitecture as well as a strong negative relationship with the connectivity gradient (Fig.3f). While we were able to reproduce the previous link between myelination and the FC gradient $27,32,49$ ( $\rho_{\text {empirical }}=-0.52, p_{\text {spin }}<0.001$ ), partial correlations of all three variables revealed that the association between myelin and the gradient loadings disappeared when controlling for ASD, whereas the ASD-myelin and ASD-gradient relationships persisted when controlling for the respective other variable. Further supporting this relationship, a hierarchical regression approach showed that ASD topology alone explained a large part of the variance in gradient loadings $\left(R_{a d j}{ }^{2}=0.57, p=4.9 e-40\right)$ and completely resolved the myelin-gradient effect in the augmented model (Fig. $3 \mathrm{~g}$ ).

These results suggest that complexity states of neural activity may underlie the link between microstructural brain features and macroscale connectivity ${ }^{32}$, bridging anatomical and functional hierarchies within the connectome. 


\section{Complexity-behavior relationships}

Lastly, as neural variability is increasingly recognized to carry functional significance $29-31,38-$ 40,51-53, we probed the behavioral implications of the observed complexity dynamics. Given the age-related reduction in drop affinity (Fig.1b) and the link between complexity drops and functional integration (Fig.3), we expected lower complexity values to reflect better behavioral performance and lower age. To test this relationship, participant age and behavioral variables were subjected to a multivariate Partial Least Squares (PLS) analysis ${ }^{63,64}$, including composite scores of crystallized and fluid abilities and the first principal components of cognitive, motor, and sensory task performance metrics (Extended Data Fig.8). PLS returned a significant latent solution on the relationship between variance in complexity and differences in behavior (permuted $p=2 e-4$ ). Confirming directional expectations, latent brain scores were positively related to latent behavioral scores $(\rho=0.28)$, and in this latent space, signal complexity was positively linked to age and negatively associated with crystallized abilities, cognitive task performance and motor function (Fig.4a). Notably, the associated bootstrap ratios showed that complexity-behavior associations were systematically constrained by functional networks $\left(x^{2}(7)=95.4, p=9.5 e-18\right)$, with strongest contributions by higher-order systems including the ventral attention and default mode network (Fig.4b). Given the direction of these complexity-behavior relationships, our findings suggest that an increased capacity for complexity drops may represent a beneficial functional profile of the brain.
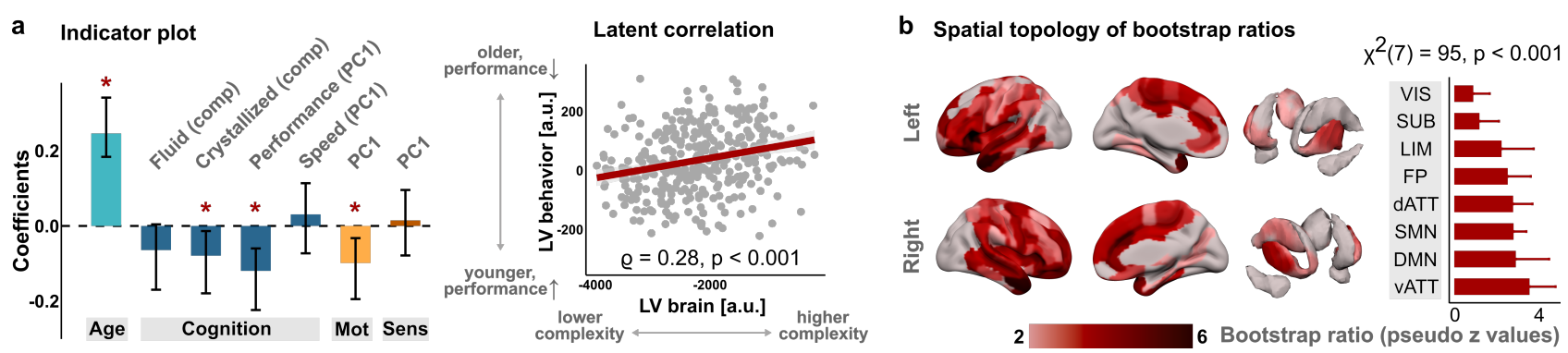

Fig. 4 | Signal complexity reflects inter-individual differences in age and behavior. a, Multivariate partial least squares correlation. Indicator plot (error bars: $95 \% \mathrm{Cl}$ ) for age and behavioral variables (comp: composite score; PC1: first principal component; *: significant coefficient). Correlation of brain and behavior scores of the first latent variable (LV). $\mathbf{b}$, Spatial topology and network distribution of bootstrap ratios. 


\section{Conclusion}

Collectively, these findings speak to a unifying principle of brain organization, grounded in a spatiotemporal complexity architecture of neural activity. This functional "human complexome" closes a gap between the variability of neural activity and the principles that govern the human connectome, including the formation of functional connections among brain regions, the propagation of activity patterns throughout the brain, as well as the functional states, economy, and hierarchy of brain networks. Our results thus point to a model of the brain in which its functional architecture unfolds from recurrent episodes of neural regularity, providing a new framework for discovery in the neurosciences.

\section{Author contributions}

SK, NS, and CF conceived the study. SK, NS, AR, and MG processed imaging data. SK designed data analysis, with feedback from NS, LW, DDG, and CF. SK developed novel analysis methods. LW and DDG contributed methodology. SK, NS, LW, AR, and MG analyzed the data. SK, NS, and AR visualized results. All authors interpreted results. SK drafted the manuscript, with contributions from NS, LW, AR, and MG. DDG and CF supervised the study. All authors revised, edited, and approved the manuscript.

\section{Acknowledgments}

SK is funded by the German Ministry of Education (BMBF grant 13GW0206D). NS is supported by a Cusanuswerk scholarship. AR receives a Mind and Brain scholarship from the Humboldt-Universität zu Berlin. MG is funded by the German Research Foundation (Deutsche Forschungsgemeinschaft, DFG; grant IRTG2150). LW and DDG are supported by an Emmy Noether Programme grant from the German Research Foundation (to DDG), and by the Max Planck UCL Centre for Computational Psychiatry and Ageing Research. CF is funded by the German Research Foundation, grant numbers 327654276 (SFB 1315), FI 2309/1-1 (Heisenberg Program), and FI 2309/2-1. The authors declare no competing interests.

\section{References}

1. Fox, M. D. et al. The human brain is intrinsically organized into dynamic, anticorrelated functional networks. Proc. Natl. Acad. Sci. U. S. A. 102, 9673 (2005).

2. Bassett, D. S. \& Sporns, O. Network neuroscience. Nat. Neurosci. 20, 353-364 (2017). 
3. Petersen, S. E. \& Sporns, O. Brain Networks and Cognitive Architectures. Neuron 88, 207-219 (2015).

4. Raichle, M. E. et al. A default mode of brain function. Proc. Natl. Acad. Sci. 98, 676 (2001).

5. Power, J. D. et al. Functional Network Organization of the Human Brain. Neuron 72, 665-678 (2011).

6. Power, J. D., Schlaggar, B. L. \& Petersen, S. E. Studying Brain Organization via Spontaneous fMRI Signal. Neuron 84, 681-696 (2014).

7. Thomas Yeo, B. T. et al. The organization of the human cerebral cortex estimated by intrinsic functional connectivity. J. Neurophysiol. 106, 1125-1165 (2011).

8. Deco, G., Jirsa, V. K. \& McIntosh, A. R. Emerging concepts for the dynamical organization of resting-state activity in the brain. Nat. Rev. Neurosci. 12, 43-56 (2011).

9. Bullmore, E. \& Sporns, O. Complex brain networks: graph theoretical analysis of structural and functional systems. Nat. Rev. Neurosci. 10, 186-198 (2009).

10. Smith, S. M. et al. Functional connectomics from resting-state fMRI. Trends Cogn. Sci. 17, 666-682 (2013).

11. Fornito, A., Zalesky, A. \& Breakspear, M. The connectomics of brain disorders. Nat. Rev. Neurosci. 16, 159-172 (2015).

12. Reid, A. T. et al. Advancing functional connectivity research from association to causation. Nat. Neurosci. 22, 1751-1760 (2019).

13. Bassett, D. S. \& Gazzaniga, M. S. Understanding complexity in the human brain. Trends Cogn. Sci. 15, 200-209 (2011).

14. Calhoun, V. D., Miller, R., Pearlson, G. \& Adalı, T. The Chronnectome: Time-Varying Connectivity Networks as the Next Frontier in fMRI Data Discovery. Neuron 84, 262-274 (2014).

15. Zalesky, A., Fornito, A., Cocchi, L., Gollo, L. L. \& Breakspear, M. Time-resolved resting-state brain networks. Proc. Natl. Acad. Sci. 111, 10341-10346 (2014).

16. Shine, J. M., Koyejo, O. \& Poldrack, R. A. Temporal metastates are associated with differential patterns of time-resolved connectivity, network topology, and attention. Proc. Natl. Acad. Sci. 113, 9888 (2016).

17. Liu, X. \& Duyn, J. H. Time-varying functional network information extracted from brief instances of spontaneous brain activity. Proc. Natl. Acad. Sci. 110, 4392-4397 (2013).

18. Allen, E. A. et al. Tracking Whole-Brain Connectivity Dynamics in the Resting State. Cereb. Cortex 24, 663-676 (2014).

19. Chang, C. \& Glover, G. H. Time-frequency dynamics of resting-state brain connectivity measured with fMRI. Neurolmage 50, 81-98 (2010).

20. Kong, X. et al. Anatomical and Functional Gradients Shape Dynamic Functional Connectivity in the Human Brain. bioRxiv 2021.03.15.435361 (2021) doi:10.1101/2021.03.15.435361.

21. Bullmore, E. \& Sporns, O. The economy of brain network organization. Nat. Rev. Neurosci. 13, 336-349 (2012).

22. Bertolero, M. A., Yeo, B. T. T., Bassett, D. S. \& D'Esposito, M. A mechanistic model of connector hubs, modularity and cognition. Nat. Hum. Behav. 2, 765-777 (2018).

23. Achard, S. \& Bullmore, E. Efficiency and Cost of Economical Brain Functional Networks. PLoS Comput. Biol. 3, e17 (2007).

24. Shafiei, G. et al. Topographic gradients of intrinsic dynamics across neocortex. eLife 9, e62116 (2020).

25. Margulies, D. S. et al. Situating the default-mode network along a principal gradient of macroscale cortical organization. Proc. Natl. Acad. Sci. 113, 12574-12579 (2016).

26. Huntenburg, J. M., Bazin, P.-L. \& Margulies, D. S. Large-Scale Gradients in Human Cortical Organization. Trends Cogn. Sci. 22, 21-31 (2018).

27. Paquola, C. et al. Microstructural and functional gradients are increasingly dissociated in transmodal cortices. PLOS Biol. 17, e3000284 (2019). 
28. Fox, M.D. \& Raichle, M. E. Spontaneous fluctuations in brain activity observed with functional magnetic resonance imaging. Nat. Rev. Neurosci. 8, 700-711 (2007).

29. Uddin, L. Q. Bring the Noise: Reconceptualizing Spontaneous Neural Activity. Trends Cogn. Sci. S1364661320301443 (2020) doi:10.1016/j.tics.2020.06.003.

30. Waschke, L., Kloosterman, N. A., Obleser, J. \& Garrett, D. D. Behavior needs neural variability. Neuron S0896627321000453 (2021) doi:10.1016/j.neuron.2021.01.023.

31. Garrett, D. D. et al. Moment-to-moment brain signal variability: A next frontier in human brain mapping? Neurosci. Biobehav. Rev. 37, 610-624 (2013).

32. Huntenburg, J. M. et al. A Systematic Relationship Between Functional Connectivity and Intracortical Myelin in the Human Cerebral Cortex. Cereb. Cortex N. Y. N 1991 27, 981-997 (2017).

33. Biswal, B., Zerrin Yetkin, F., Haughton, V. M. \& Hyde, J. S. Functional connectivity in the motor cortex of resting human brain using echo-planar MRI. Magn. Reson. Med. 34, 537-541 (1995).

34. Zalesky, A., Fornito, A. \& Bullmore, E. On the use of correlation as a measure of network connectivity. Neurolmage 60, 2096-2106 (2012).

35. Zamani Esfahlani, F. et al. High-amplitude cofluctuations in cortical activity drive functional connectivity. Proc. Natl. Acad. Sci. 202005531 (2020) doi:10.1073/pnas.2005531117.

36. Pope, M., Fukushima, M., Betzel, R. \& Sporns, O. Modular origins of high-amplitude cofluctuations in fine-scale functional connectivity dynamics. http://biorxiv.org/lookup/doi/10.1101/2021.05.16.444357 (2021) doi:10.1101/2021.05.16.444357.

37. Baracchini, G. et al. Inter-regional BOLD signal variability is an organizational feature of functional brain networks. Neurolmage 118149 (2021) doi:10.1016/j.neuroimage.2021.118149.

38. Garrett, D. D., Kovacevic, N., McIntosh, A. R. \& Grady, C. L. Blood Oxygen Level-Dependent Signal Variability Is More than Just Noise. J. Neurosci. 30, 4914 (2010).

39. Garrett, D. D., Kovacevic, N., McIntosh, A. R. \& Grady, C. L. The Importance of Being Variable. J. Neurosci. 31, 4496 (2011).

40. Guitart-Masip, M. et al. BOLD Variability is Related to Dopaminergic Neurotransmission and Cognitive Aging. Cereb. Cortex 26, 2074-2083 (2016).

41. Vézquez-Rodríguez, B., Liu, Z.-Q., Hagmann, P. \& Misic, B. Signal propagation via cortical hierarchies. Netw. Neurosci. 4, 1072-1090 (2020).

42. Yousefi, B. \& Keilholz, S. Propagating patterns of intrinsic activity along macroscale gradients coordinate functional connections across the whole brain. Neurolmage 231, 117827 (2021).

43. Amemiya, S., Takao, H., Hanaoka, S. \& Ohtomo, K. Global and structured waves of rs-fMRI signal identified as putative propagation of spontaneous neural activity. Neurolmage 133, 331340 (2016).

44. Mitra, A., Snyder, A. Z., Tagliazucchi, E., Laufs, H. \& Raichle, M. E. Propagated infra-slow intrinsic brain activity reorganizes across wake and slow wave sleep. eLife 4, e10781 (2015).

45. Newbold, D. J. et al. Plasticity and Spontaneous Activity Pulses in Disused Human Brain Circuits. Neuron S0896627320303536 (2020) doi:10.1016/j.neuron.2020.05.007.

46. Raut, R. V. et al. Organization of Propagated Intrinsic Brain Activity in Individual Humans. Cereb. Cortex 30, 1716-1734 (2020).

47. Mitra, A. \& Raichle, M. E. How networks communicate: propagation patterns in spontaneous brain activity. Philos. Trans. R. Soc. B Biol. Sci. 371, 20150546 (2016).

48. Glasser, M. F. \& Van Essen, D. C. Mapping human cortical areas in vivo based on myelin content as revealed by T1- and T2-weighted MRI. J. Neurosci. Off. J. Soc. Neurosci. 31, 1159711616 (2011).

49. Vos de Wael, R. et al. BrainSpace: a toolbox for the analysis of macroscale gradients in neuroimaging and connectomics datasets. Commun. Biol. 3, 103 (2020). 
50. Suárez, L. E., Markello, R. D., Betzel, R. F. \& Misic, B. Linking Structure and Function in Macroscale Brain Networks. Trends Cogn. Sci. 24, 302-315 (2020).

51. Nomi, J. S., Bolt, T. S., Ezie, C. E. C., Uddin, L. Q. \& Heller, A. S. Moment-to-Moment BOLD Signal Variability Reflects Regional Changes in Neural Flexibility across the Lifespan. J. Neurosci. 37, 5539 (2017).

52. Garrett, D. D. et al. Amphetamine modulates brain signal variability and working memory in younger and older adults. Proc. Natl. Acad. Sci. 112, 7593 (2015).

53. Armbruster-Genç, D. J. N., Ueltzhöffer, K. \& Fiebach, C. J. Brain Signal Variability Differentially Affects Cognitive Flexibility and Cognitive Stability. J. Neurosci. 36, 3978 (2016).

54. Van Essen, D. C. et al. The WU-Minn Human Connectome Project: An overview. Neurolmage 80, 62-79 (2013).

55. Glasser, M. F. et al. A multi-modal parcellation of human cerebral cortex. Nature 536, 171178 (2016).

56. Fadlallah, B., Chen, B., Keil, A. \& Príncipe, J. Weighted-permutation entropy: A complexity measure for time series incorporating amplitude information. Phys. Rev. E 87, 022911 (2013).

57. Bandt, C. \& Pompe, B. Permutation entropy: a natural complexity measure for time series. Phys. Rev. Lett. 88, 174102 (2002).

58. Garrett, D. D., Epp, S. M., Perry, A. \& Lindenberger, U. Local temporal variability reflects functional integration in the human brain. Neurolmage 183, 776-787 (2018).

59. Fan, L. et al. The Human Brainnetome Atlas: A New Brain Atlas Based on Connectional Architecture. Cereb. Cortex 26, 3508-3526 (2016).

60. McNab, J. A. et al. The Human Connectome Project and beyond: initial applications of 300 $\mathrm{mT} / \mathrm{m}$ gradients. Neurolmage 80, 234-245 (2013).

61. Ramirez-Mahaluf, J. P. et al. Transitions between human functional brain networks reveal complex, cost-efficient and behaviorally-relevant temporal paths. Neurolmage 219, 117027 (2020).

62. Alexander-Bloch, A. F. et al. On testing for spatial correspondence between maps of human brain structure and function. Neurolmage 178, 540-551 (2018).

63. Krishnan, A., Williams, L. J., Mclntosh, A. R. \& Abdi, H. Partial Least Squares (PLS) methods for neuroimaging: a tutorial and review. Neuroimage 56, 455-475 (2011).

64. Mclntosh, A., Bookstein, F., Haxby, J. V. \& Grady, C. Spatial pattern analysis of functional brain images using partial least squares. Neuroimage 3, 143-157 (1996). 


\section{Online methods}

\section{Data and preprocessing}

Data were obtained from the Human Connectome Project $(\mathrm{HCP})^{54,65}$, including minimally processed resting-state $\mathrm{fMRI}^{65,66}$, diffusion-weighted images (DWI), T1-/T2 image ratios ${ }^{48}$ as well as demographics and behavioral scores from 343 participants (138 males, 205 females). MRI data were acquired in a 3T scanner at Washington University in St. Louis with multiband echo-planar imaging (1200 volumes, TR $=0.72 \mathrm{~s}, 2 \mathrm{~mm}$ isotropic voxels). Two runs, each with one right-to-left and one left-to-right phase encoding protocol, were acquired for each participant on two consecutive days. These two runs in a session were averaged, resulting in two resting-state recordings of approximately 15 minutes for each participant (1200 sampled time points). Two scans from different participants were excluded because of incomplete data, resulting in a total of 684 scans. All acquisition parameters and processing pipelines are described in detail in refs ${ }^{65-69}$. We manually applied bandpass filtering [0.01 Hz to $0.1 \mathrm{~Hz}$ ] and z-scoring to each voxel before extracting the BOLD timeseries based on the Brainnetome (BNA) atlas ${ }^{59}$, which includes 246 cortical and subcortical regions of interest (ROI). Assignment of ROls to seven canonical resting-state networks (visual (VIS), somatomotor (SMN), dorsal attention (dATT), ventral attention (VATT), default mode (DMN), frontoparietal (FP), and limbic (LIM)) is provided with the BNA template ${ }^{59}$. This mapping is derived from the cortical network parcellation by Yeo et al. ${ }^{70}$ and is available from www.brainnetome.org/resource. Regions 165, 177, and 178 (located in the left insular gyrus as well as left and right cingulate) are not labelled in the template and were manually assigned to the frontoparietal network based on their overlap with the Yeo parcellation. Furthermore, regions in the basal ganglia, thalamus, hippocampus, and amygdala were subsumed as subcortical parcels. Finally, to investigate the sensitivity of the observed complexity dynamics to higher and lower spatial granularity, the atlases provided by Shen et al. ${ }^{71}$ (268 ROls) and Shirer et al. ${ }^{72}$ (90 ROls) were included for comparative parcellation, with highly convergent results (Extended Data Fig.4-5).

\section{Time-resolved estimation of signal complexity}

Signal complexity of the BOLD timeseries was calculated as weighted permutation entropy ${ }^{56}$ (WPE) through symbolic encoding of the timeseries vectors. WPE is an amplitude-sensitive extension of permutation entropy $(\mathrm{PE})^{57}$, an information-theoretic quantity that captures the 
degree of irregularity as the Shannon entropy ${ }^{73}$ on the occurrence of symbolic motifs within a timeseries.

WPE is defined as

$$
H_{W P E}(\mathrm{~m}, \tau)=-\sum_{i: \pi_{i}^{m, \tau} \in \Pi} p_{w}\left(\pi_{i}^{m, \tau}\right) \ln p_{w}\left(\pi_{i}^{m, \tau}\right)
$$

where $\mathrm{m}$ is the length of symbolic motifs in the timeseries, $\tau$ is a lag parameter indicating the number of time points to shift along the timeseries, $\pi_{i}^{m, \tau}$ represents the $i$ 'th symbolic motif out of the set of possible motifs $\Pi$ given the motif length, and $p_{w}\left(\pi_{i}^{m, \tau}\right)$ is the varianceweighted relative frequency of motif $i$, as detailed in ref ${ }^{56}$. Following methodological considerations $^{56,74}$ and previous applications of WPE to neural data analysis ${ }^{75,76}$, a motif length of $\mathrm{m}=3$ and a lag parameter of $\tau=1$ was applied to compute the motif distribution over the signal vector of interest, and WPE bit values were normalized to lie in $[0,1]^{56}$. Notably, by leveraging both pattern diversity (assessed by PE) and amplitude information (assessed by the standard deviation over the BOLD vector), WPE captures signal dynamics that remain undetected when considering only one of these characteristics (Extended Data Fig.1-2). A further advantage of WPE lies in its ability to accommodate a time-resolved approach, based on the comparatively low number of timeseries samples needed to achieve stable estimation ${ }^{74,77}$. Accordingly, we here applied a sliding window approach, where the entire BOLD timeseries in divided into temporally contiguous windows of a prespecified length and overlap. In the main text, we report the findings for a window length of 60 TRs (43.2 seconds) with 95\% overlap, yielding a window-to-window temporal resolution of 3 TRs (2.16 seconds) and a time-resolved signal complexity vector of 380 windows. To investigate the sensitivity of the observed complexity dynamics to the windowing parameters, we analyzed window lengths of 60,90 , and 120 samples with $95 \%$ and $90 \%$ window overlap, respectively, with highly convergent results (Extended Data Fig.4).

Based on the ubiquitously observed pattern of predominant high complexity with recurrent complexity drops, we defined a threshold of drop engagement as the first percentile of the total WPE distribution (critical WPE $=0.273$ ). Accordingly, whenever a region's signal complexity met this value for a given BOLD window, it was counted as engaged in a complexity drop in that window (Fig.1a). Notably, whether this thresholding procedure was applied across all participants or as participant-specific percentile thresholds made essentially no difference to the spatial distribution of where complexity drops occurred $(r=$ $0.99, p<0.001)$. We then investigated to which extent brain regions coincide in their drop 
engagement over time. To this end, we iterated over all BOLD windows in the data set and counted those regions that met the drop threshold simultaneously in each window. This count matrix was normalized by the maximum coincidence count, yielding drop coincidence values from 0 to 1 . The pairwise drop coincidences between any two regions were then subjected to correlation analyses with the corresponding functional connectivity values (Fig.1c). To investigate how the likelihood to engage in complexity drops is distributed over brain regions and age groups, we furthermore calculated time-resolved drop affinity for each scan. To this end, a binary matrix (BOLD windows over ROIs) was created, containing ones whenever a region's signal complexity met the drop threshold in a particular window, and zero otherwise. These affinity matrices were then averaged to estimate participant-wise and region-wise drop affinity. Note that the time-resolved affinity vector for individual scans also served the observation of brain-wide engagement in complexity drops (Fig.2a) and the subsequent propagation analysis.

We furthermore investigated the degree of similarity between a region's complexity timeseries with its contralateral equivalent (Fig.1d). To this end, we defined an interhemispheric symmetry index (ISI), which is computed as the correlation coefficient of a region with its contralateral equivalent, weighted by the proportion of the 246 BNA regions that were less strongly correlated with that region. Accordingly, a region with high crosshemispheric similarity shows many regions that are less correlated with it than the contralateral equivalent, yielding a weighting factor close to one. In contrast, if there are many regions that exhibit a higher correlation to the target region than the contralateral ROI, this weighting factor decreases, resulting in lower ISI values.

\section{Descriptive statistics}

Group-level comparisons (e.g., by age or by resting-state networks) were carried out with the nonparametric Kruskal-Wallis test ${ }^{78}$, which rests on observation ranks and applies to $\mathrm{n}>2$ groups. Significant group differences at $\alpha=0.05$ were followed up by pairwise rank sum tests $^{79}$, with adjustment for multiple comparisons using the false discovery rate (FDR). Associations between continuous variables were assessed with parametric or nonparametric correlation tests, depending on the underlying distributions. Participant age was given in the age groups of 22-25, 26-30, 31-35, and >35 years; for age-wise comparisons, however, participants over 35 years were excluded, as this only applied to $\mathrm{n}=3$ individuals. For all assessments of directional effects, two-tailed tests were applied. 


\section{Propagation analysis}

The propagation of complexity drops across the brain was formalized as a graph theoretical problem. Here, each individual propagation was modelled as a directed graph where nodes represent brain regions engaged in complexity drops and directed edges represent the progression in time from one BOLD window to the next.

To identify drop cascades in the dataset, a stepwise search procedure was applied. First, all BOLD windows with at least $n=10$ regions simultaneously engaged in complexity drops were identified in the time-resolved affinity vector. If there were several temporally contiguous windows that met this criterion, the window with the maximum number of engaged regions was defined as the peak layer in the directed graph. From this peak layer, a window-by-window backward and forward search identified the neighboring windows in which the number of engaged regions increased until reaching the peak layer (propagation phase) and decreased after the peak (fade phase), respectively. The minimum cascade length thus included three contiguous windows (initialization - peak - fade). Although very rare, instances in which the windows directly before or after the peak showed no engaged regions were discarded. To investigate the spread of complexity drops in the propagation phase, individual directed graphs were constructed from the initialization window - in which the engaged regions represent the graph's source nodes - to the peak window (Fig.2b).

For all windows from initialization to peak, the newly engaging regions from one window to the next were registered to compute the empirical transition probability, where all newly engaged regions in window $i$ obtain directed edges to all newly engaging regions in window $i+1$. The region-by-region transition probability matrix (TPM) was then constructed based on the path-weighted edges in the propagation graph. Here, edges in temporally contiguous windows were assigned a weight of 1 , while connections in non-neighboring windows were assigned the inverse of the path length (e.g., $1 / 2$ for connections from window $i$ to window $i+2$ ) to account for the temporal evolution of the spread. For instance, if engagement of a region $A$ is frequently followed by engagement of region $B$, but through variable intermediate engagement of regions $C, D$, etc., this is missed in a binary TPM, as only neighboring window are considered. Construction of propagation graphs and TPMs over individual cascades subsequently allowed for participant-wise and group-level analyses: to capture cascade origination, the source node probability of each brain region was computed as the rate of occurrence in the initialization window. The diversity of source nodes was calculated as the percentage of unique brain regions ever initializing a participant's cascades. The average TPM over individual spreads yielded the directed group-level propagation network 
in Fig.2e, representing the $95^{\text {th }}$ percentile of path-weighted transition probabilities. Graph construction and topological analyses on the propagation network was implemented with the igraph package (version 1.2.5) for $R^{80}$. The hub structure of the network was quantified as Kleinberg centrality, an extension of eigenvector centrality for directed networks ${ }^{81}$. Geodesic distances were computed for every node-to-node comparison in the propagation network and averaged for all combinations within and across resting-state networks. The average distance matrix was then subjected to hierarchical clustering using the default complete linkage method ${ }^{82}$. As this approach revealed a highly suggestive order from unimodal to transmodal networks, we explicitly investigated this cluster structure by defining a unimodal and a transmodal cluster group, subsequently tested with a Monte Carlo simulation using the sigclust package ${ }^{83}$. The null hypothesis of this cluster test is that the data emanate from a single Gaussian distribution. To assess this hypothesis, 5000 Gaussian samples were created to estimate the distribution of the cluster index ${ }^{83}$, a test statistic defined as the sum of within-class sums of squares about the mean in relation to the total sum of squares about the overall mean (cf., https://rdrr.io/cran/sigclust/man).

\section{Clustering of complexity states}

The time-resolved signal complexity matrix (rows: 684 scans $\times 380$ windows $=259920$ observations; columns: 246 ROIs) was subjected to unsupervised structure detection to investigate temporally discrete complexity states. To this end, we applied the MATLABinbuilt k-means clustering algorithm with a maximum of 1000 iterations, 20 replicates with random initial positions to avoid local minima, the k-means ++ heuristic for centroid initialization, and the squared Euclidean distance as the target metric to be optimized.

Based on the observed complexity dynamics (Fig.1a; online repository: https://osf.io/mr8f7), we expected a predominant high-complexity state, an infrequent low-complexity state, and a varying number of intermediate complexity states. As common cluster evaluation indices to determine the optimal $k$ of expected clusters resulted in heterogeneous estimates between $\mathrm{k}=3$ and $\mathrm{k}=5$ depending on windowing parameters and the optimization criterion applied, we specified k=4 (i.e., two intermediate complexity states) and ran comprehensive control analyses for $k=3$ (i.e., one intermediate state) and $k=5$ (i.e., three intermediate states). Results reported in the main text were extremely robust against the choice of $k$, including the occupancy-complexity relationship, the impact of complexity states on network strength and topology, state dispersion, as well as the spatial topology of complexity states (whose spatial heterogeneity was assessed based on the modified likelihood ratio test ${ }^{84}$, 
MLRT) and its link to myelination and the primary functional connectivity gradient (Extended Data Fig. 6-7).

\section{State dispersion index (SDI)}

To assess the degree to which the empirical state visits of an individual participant were distributed across the $k$ possible complexity states, we defined a state dispersion index (SDI), calculated as

$$
S D I=1-\frac{W S D_{\text {empirical }}}{W S D_{\text {maximum }}}
$$

where WSD corresponds to the first Wasserstein distance between two discrete distributions ${ }^{85}$. In particular, $W S D_{\text {empirical }}$ is given by the distance between the empirically observed state occupancy $(F i g .3 a+c)$ and theoretical maximum dispersion (i.e., a perfect uniform distribution). This distance is normalized by $W S D_{\text {maximum }}$, which is given by the distance between the theoretical minimum dispersion (only one of $k$ states was ever visited) and the uniform distribution. Consequently, the $S D I$ lies in $[0,1]$, and is bounded by 0 if only one state was ever visited in the empirical state occupancy (minimum dispersion), and 1 if the latter is identical to the uniform (maximum dispersion).

\section{Across-state distance (ASD)}

To characterize the spatial topology across the estimated complexity states, we defined an index of across-state distance (ASD). Let $K$ denote the set of unique centroid pairs $(i, j)$ in $k$-dimensional state space. For each brain region, the ASD is computed as the cumulative Euclidean distance $D$ over all pairs of centroids $C$ in $K$ :

$$
A S D=\sum_{(i, j) \in K} D\left(C_{i} \| C_{j}\right) .
$$

For the Brainnetome parcellation, this yields a $1 \times 246$ vector, where higher values indicate brain regions that show greater centroid-to-centroid distances and thus more pronounced differences in signal complexity over the estimated complexity states. Note that the resulting ASD topology was very robust against the choice of $k$ (Extended Data Fig.6-7), and that this vector constituted the input for the subsequent analyses linking state topology to cortical myelination and the primary functional connectivity gradient. 


\section{Functional connectivity and network topology}

Static functional connectivity was estimated as the ROI-by-ROI Pearson correlation matrix over the entire resting-state recording. These matrices were averaged over runs and participants to obtain the group-level functional connectivity matrix for investigating the association to drop coincidence and for the construction of the macroscale connectivity gradient (see below). Furthermore, dynamic functional connectivity was computed as the correlation matrix over each BOLD window, resulting in a $246 \times 246 \times 380$ array for the specified window parameters. Dynamic connectivity strength was calculated as the mean over these window-wise matrices, yielding a 1x380 vector. The topology of the time-resolved functional networks was assessed with the Brain Connectivity Toolbox ${ }^{86}$, available from www.brain-connectivity-toolbox.net. Network modularity as a measure of functional segregation was estimated through Louvain community detection (community_louvain.m) with asymmetric treatment of negative weights as recommended in ref. ${ }^{86}$ and with a $\mathrm{Y}$ parameter of 1.05 to accommodate the ability to detect smaller modules ${ }^{86}$. Furthermore, global efficiency as an estimate of functional integration was computed using the efficiency_wei.m function, with negative weights discarded ${ }^{86}$. This approach yielded vectors of time-resolved modularity and global efficiency (1×380 BOLD windows) for each restingstate recording. These time-resolved vectors constituted the input for the calculation of both the participant averages (Fig.3a) and the window-to-window transitions (Fig.3b) of network descriptors, where the corresponding complexity state vector from the clustering output served as a state-wise mask.

\section{Structural connectivity estimation}

Structural connectivity matrices were computed using probabilistic tractography as implemented in the FMRIB's Diffusion Toolbox ProbtrackX GPU program ${ }^{87}$. Diffusionweighted data provided as the "Diffusion BedpostX" package were available for 340 of the 343 participants in the study population. These data were preprocessed as described in ref ${ }^{65}$, including registration to native space, movement and eddy currents correction, and application of BedpostX to model white matter fiber orientations for probabilistic tractography. The connectivity distribution is then computed using iterations of streamlines propagated from seed regions to target regions. Briefly, a single propagation entails moving along a streamline by steps of a specified length, evaluating for exclusion or termination criteria at each step, and continuing this process until criteria for exclusion (streamline discarded) or termination (propagation ended but streamline retained) are met. The output 
is a matrix which quantifies the number of non-discarded streamlines between a seed and target.

In line with the functional analyses, BNA regions were specified as seed ROls to guide tractography. Network mode was applied in ProbtrackX to compute a ROI-by-ROI connectivity matrix, where rows represent seed ROIs and columns correspond to target ROls. Each BNA region was input as a binary mask in MNI space. Since the seed ROls (standard) and processed diffusion data (native) were not registered to the same space, bidirectional participant-specific nonlinear transformations between standard and structural space as provided by the HCP were passed to ProbtrackX. Following the recommendation that the inclusion of additional anatomical priors increases the biological plausibility of modelled white matter tracts ${ }^{88}$, we included participant-specific gray matter (GM) masks from Freesurfer as termination masks (--stop). By terminating a propagated streamline as soon as it enters this GM mask, streamlines are forced to terminate near the boundary between GM and WM instead of travelling further into a GM region. Streamline termination parameters were applied as ProbtrackX defaults: streamline curvature threshold exceeded (0.2, $~ 80$ degrees), streamline path returns to a point which it already intersected previously (--loopcheck), streamline exits the nodiff_brain_mask (BedpostX output), and maximum number of steps per streamline (2000) reached using a step length of $0.5 \mathrm{~mm}$.

For each voxel in a seed region, 1000 samples were propagated. Distance correction (--pd) was applied to compensate for the bias that the probability of a streamline successfully reaching the target $\mathrm{ROI}$ decreases as the distance between a seed and target $\mathrm{ROI}$ increases. Distance correction as implemented in ProbtrackX adjusts the connectivity distribution between a seed-target ROI pair by multiplying the number of successful streamlines between the ROls by the average path length of the streamlines. Finally, ROIby-ROI connectivity matrices were then normalized using the probabilistic streamline connectivity index (PSCI) procedure, as described in ref ${ }^{60}$. The $\mathrm{PSCl}$ scales the streamline counts between ROI pairs based on the number of propagated and successful streamlines as well as the size of both the seed and target ROls.

\section{Myelination estimation}

Cortical grayordinate myelin maps obtained from the $T 1 w / T 2 w$ image ratios ${ }^{48}$ were used to calculate the average myelin content of ROls in the atlas template. Since our parcellation approach did not benefit from the higher resolution of the $164 \mathrm{k}$ images, we analyzed the unsmoothed, bias-corrected $32 \mathrm{k}$ areal-feature-based aligned images 
(“MyelinMap_BC_MSMAll.32k_fs_LR.dscalar.nii”) using the Multimodal Surface Matching algorithm $^{89}$. Parcel-wise myelin content was obtained using the Connectome Workbench (command: -cifti-parcellate) by averaging over the myelin map within the respective BNA regions.

\section{Gradient construction, spin permutation, and hierarchical regression}

The macroscale connectivity gradient was computed on the group-average functional connectivity matrix. As only the cortex is mapped to the FreeSurfer sphere for later spin permutation, the input matrix was restricted to the $210 \times 210$ cortical ROls of the BNA. Gradient analysis was implemented with the BrainSpace toolbox ${ }^{49}$ for neuroimaging and connectomics datasets. Following ref. ${ }^{25}$, cosine similarity was applied to compute the affinity matrix. A total of 10 components, diffusion embedding for non-linear dimensionality reduction (diffusion time of 0 , alpha parameter of 0.5 ), and $90 \%$ region-wise feature sparsity were used for gradient construction, following the default recommendations. ${ }^{49}$ The gradient fit on these data is displayed in Fig.3f of the main text and precisely identified the canonical unimodal to transmodal connectivity gradient first reported in ref. ${ }^{25}$ To examine the association between these gradient loadings and the other cortical features (myelination, across-state distance), we applied spin permutation correlation of the respective cortical maps. This approach consists in a series of random spherical rotations that preserve the spatial autocorrelation of the data, resulting in empirical null models that counteract the potential inflation of statistical significance in simple univariate tests ${ }^{49,62,90}$. Here we implemented a variant of this procedure that specifically applies to parcellated instead of vertex-wise cortical maps ${ }^{91}$. Nonparametric correlations over 5000 random rotations were computed to estimate the empirical distribution of the test statistic for the three comparisons between gradient loadings, cortical myelination, and the across-state distance.

The empirically observed associations across these cortical features were furthermore subjected to a nonparametric partial correlation analysis using the ppcor package for $\mathrm{R}^{92}$ (Fig.4g). As this approach suggested high explanatory power of complexity topology, these analyses were corroborated with a hierarchical regression approach, where variance in gradient loadings was modelled as a function of cortical myelination (compact model 1), across-state-distance (compact model 2), or both (augmented model 3). These individual linear models were then compared through F-tests for nested models with the ImSupport package for R (https://cran.r-project.org/web/packages/lmSupport/lmSupport.pdf). 


\section{Complexity-behavior associations}

To assess associations between signal complexity and participant characteristics, average WPE values per ROI and participant were tested against age and indices of cognitive performance, motor skills as well as sensory scores, as provided with the HCP data. To ensure data quality and minimize the impact of non-normally distributed variables, we transformed response time data to speed via inversion, subjected skewed data to log transformations (if absolute skewness $>1$ ), excluded data from participants with missing values in at least one variable, and removed outliers (mean \pm 4 standard deviations). In total, data from 13 participants were thus excluded, leaving $n=330$ participants for behavioral analyses. To this end, we tested the multivariate relationship between BOLD signal complexity and individual participant measures by means of a partial least squares (PLS) analysis $^{63,64,93}$ (Fig.4). We applied a behavioral PLS approach that allows for the estimation of multivariate correlations between a three-dimensional brain variable (average WPE per ROI and participant) and multiple behavioral measures (Extended Data Fig.8). To focus this analysis on general features of behavioral and cognitive abilities, we performed principal component analyses (PCA) on the respective sets of single measures of performance and speed in cognitive tasks, motor performance, and sensory skills. For each of the four domains, the first principal component accounted for more than $35 \%$ of total variance and component loadings were strictly positive, resulting in four principal component scores per participant that entered PLS analyses alongside individual age as well as composite scores of fluid and crystalized cognition, as provided with the HCP data, such that seven behavioral variables were used in the PLS analysis.

In brief, PLS works via the calculation of a correlation matrix that captures the betweenparticipant correlation of the target brain measure in each region and the behavioral metrics of interest (matrix size: $N_{\text {regions }} \times \mathrm{N}_{\text {behavior }}=246 \times 7$ ). Next, this rank-correlation matrix is decomposed using singular value decomposition (SVD), resulting in $\mathrm{N}_{\text {behavior }} \times \mathrm{N}_{\text {behavior latent }}$ variables. This approach produces two main outputs: (1) a singular value for every latent variable, indicating the proportion of cross-block variance explained by the latent variable, and (2) a pattern of weights ( $\left.\mathrm{N}_{\text {regions }}\right)$ representing the rank correlation strength between WPE and behavioral measures. The multiplication (dot product) of these weights with region-wise WPE yields brain scores reflecting the between-participant correlation of complexity and behavioral metrics. Statistical significance of these brain scores and underlying latent variables was tested by permuting behavioral measures across participants and recalculating the singular value of each latent variable (5000 permutations). 
To furthermore estimate the robustness of the calculated weights, a bootstrap procedure was applied (5000 bootstraps with replacement). The division of the empirical weights by the bootstrapped standard error yields bootstrap ratios (BSR). These BSR values estimate the robustness of observed effects on a region-wise level and can be interpreted as values from a z-distribution. Hence, BSR values exceeding 1.96 relate to a correlation between the latent brain scores (weighted average WPE) and the latent behavioral scores (weighted behavioral metrics) at a $p$-value of $p<0.05$, and likewise $p<0.01$ for $B S R$ values exceeding 2.7. Finally, bootstrap resampling was also applied to estimate the $95 \%$ confidence intervals for the observed indicator correlations between WPE and behavioral measures.

\section{Data and code availability}

Analyses were implemented in bash, MATLAB (The MathWorks Inc., Natick, Massachusetts, version $2019 \mathrm{~b}$ ), and $\mathrm{R}^{82}$ (version 3.6.3). Input data and code supporting the findings presented here will be made available upon acceptance of the manuscript.

\section{References}

65. Glasser, M. F. et al. The minimal preprocessing pipelines for the Human Connectome Project. Neurolmage 80, 105-124 (2013).

66. Salimi-Khorshidi, G. et al. Automatic denoising of functional MRI data: Combining independent component analysis and hierarchical fusion of classifiers. Neurolmage 90, 449-468 (2014).

67. Jenkinson, M., Beckmann, C. F., Behrens, T. E. J., Woolrich, M. W. \& Smith, S. M. FSL. Neurolmage 62, 782-790 (2012).

68. Fischl, B. FreeSurfer. Neurolmage 62, 774-781 (2012).

69. Jenkinson, M., Bannister, P., Brady, M. \& Smith, S. Improved Optimization for the Robust and Accurate Linear Registration and Motion Correction of Brain Images. Neurolmage 17, 825841 (2002).

70. Yeo, B. T. et al. The organization of the human cerebral cortex estimated by intrinsic functional connectivity. J. Neurophysiol. (2011).

71. Shen, X., Tokoglu, F., Papademetris, X. \& Constable, R. T. Groupwise whole-brain parcellation from resting-state fMRI data for network node identification. Neurolmage 82, 403415 (2013).

72. Shirer, W. R., Ryali, S., Rykhlevskaia, E., Menon, V. \& Greicius, M. D. Decoding subjectdriven cognitive states with whole-brain connectivity patterns. Cereb. Cortex N. Y. N 199122 , 158-165 (2012).

73. Shannon, C. E. A mathematical theory of communication. Bell Syst. Tech. J. 27, 379-423 (1948).

74. Riedl, M., Müller, A. \& Wessel, N. Practical considerations of permutation entropy. Eur. Phys. J. Spec. Top. 222, 249-262 (2013). 
75. Waschke, L., Wöstmann, M. \& Obleser, J. States and traits of neural irregularity in the agevarying human brain. Sci. Rep. 7, 17381 (2017).

76. Waschke, L., Tune, S. \& Obleser, J. Local cortical desynchronization and pupil-linked arousal differentially shape brain states for optimal sensory performance. eLife 8, e51501 (2019).

77. Amigó, J., Zambrano, S. \& Sanjuán, M. A. Combinatorial detection of determinism in noisy time series. EPL Europhys. Lett. 83, 60005 (2008).

78. Kruskal, W. H. \& Wallis, W. A. Use of ranks in one-criterion variance analysis. J. Am. Stat. Assoc. 47, 583-621 (1952).

79. Wilcoxon, F. Individual Comparisons by Ranking Methods. Biom. Bull. 1, 80 (1945).

80. Csardi, G. \& Nepusz, T. The igraph software package for complex network research. InterJournal Complex Syst. 1695, 1-9 (2006).

81. Kleinberg, J. M. Authoritative sources in a hyperlinked environment. J. ACM JACM 46, 604632 (1999).

82. R Core Team. R: A Language and Environment for Statistical Computing. (R Foundation for Statistical Computing, 2020).

83. Liu, Y., Hayes, D. N., Nobel, A. \& Marron, J. S. Statistical significance of clustering for highdimension, low-sample size data. J. Am. Stat. Assoc. 103, 1281-1293 (2008).

84. Krishnamoorthy, K. \& Lee, M. Improved tests for the equality of normal coefficients of variation. Comput. Stat. 29, 215-232 (2014).

85. Kolouri, S., Park, S., Thorpe, M., Slepčev, D. \& Rohde, G. K. Optimal Mass Transport: Signal processing and machine-learning applications. IEEE Signal Process. Mag. 34, 43-59 (2017).

86. Rubinov, M. \& Sporns, O. Complex network measures of brain connectivity: Uses and interpretations. Neurolmage 52, 1059-1069 (2010).

87. Hernandez-Fernandez, M. et al. Using GPUs to accelerate computational diffusion MRI: From microstructure estimation to tractography and connectomes. Neurolmage 188, 598-615 (2019).

88. Yeh, C., Jones, D. K., Liang, X., Descoteaux, M. \& Connelly, A. Mapping Structural Connectivity Using Diffusion MRI: Challenges and Opportunities. J. Magn. Reson. Imaging jmri.27188 (2020) doi:10.1002/jmri.27188.

89. Robinson, E. C. et al. MSM: A new flexible framework for Multimodal Surface Matching. Neurolmage 100, 414-426 (2014).

90. Alexander-Bloch, A., Raznahan, A., Bullmore, E. \& Giedd, J. The convergence of maturational change and structural covariance in human cortical networks. J. Neurosci. 33, 28892899 (2013).

91. Váša, F. et al. Adolescent Tuning of Association Cortex in Human Structural Brain Networks. Cereb. Cortex 28, 281-294 (2018).

92. Kim, S. ppcor: an R package for a fast calculation to semi-partial correlation coefficients. Commun. Stat. Appl. Methods 22, 665 (2015).

93. Mclntosh, A. R. \& Lobaugh, N. J. Partial least squares analysis of neuroimaging data: applications and advances. Neuroimage 23, S250-S263 (2004). 


\section{Extended Data Figures}

a Sinusoid signal with constant frequency and amplitude variation
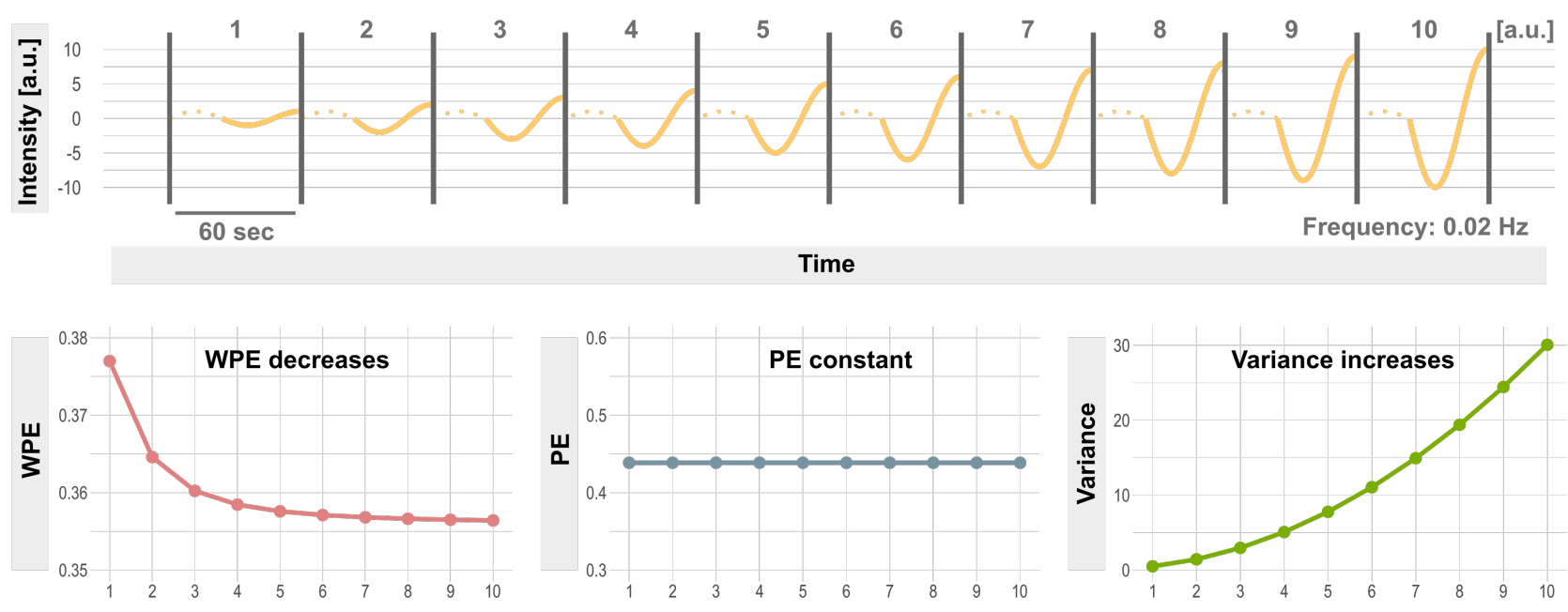

Amplitude change [a.u.]

b

Sinusoid signal with constant amplitude and frequency variation
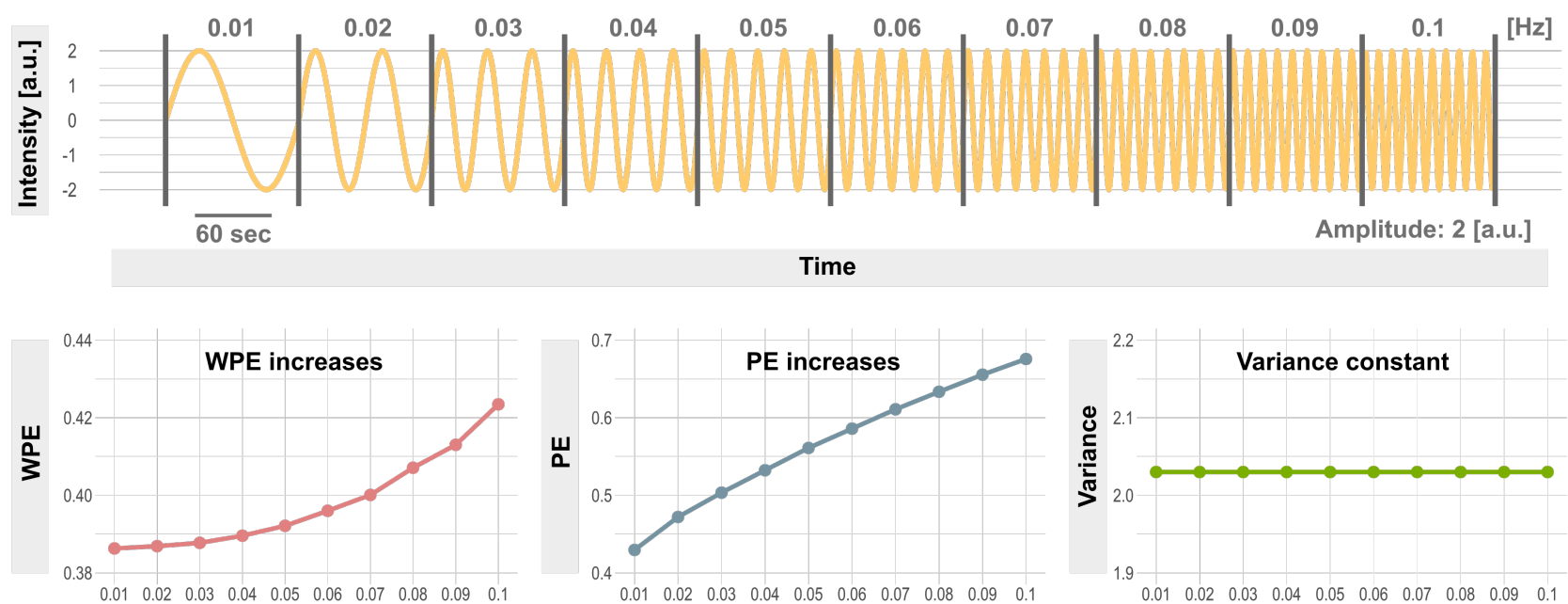

Frequency $[\mathrm{Hz}]$

Extended Data Fig.1 | Weighted permutation entropy reflects amplitude and frequency variation that remains undetected when considering only pattern diversity or signal variance alone. a, Simulated sinusoid signal with constant frequency in the range of real BOLD signals $(0.02$ $\mathrm{Hz}$ ) and with systematically increasing amplitude variation that results in changes of weighted permutation entropy (WPE) and amplitude variance, although pattern diversity alone (permutation entropy, PE) remains identical. b, Simulated sinusoid signal with constant amplitude and systematically increasing frequency variation that results in changes of weighted permutation entropy (WPE) and pattern diversity (PE), although signal variance remains unchanged. Note that WPE values monotonically increase with the higher frequency content, reaching about 0.42 at 0.1 $\mathrm{Hz}$ (identical to the applied low-pass filter settings in BOLD preprocessing), consistent with the empirically observed upper limit of the complexity timeseries derived from real BOLD signals (Fig.1a; online repository: https://osf.io/mr8f7; Extended Data Fig.3-4). 
a
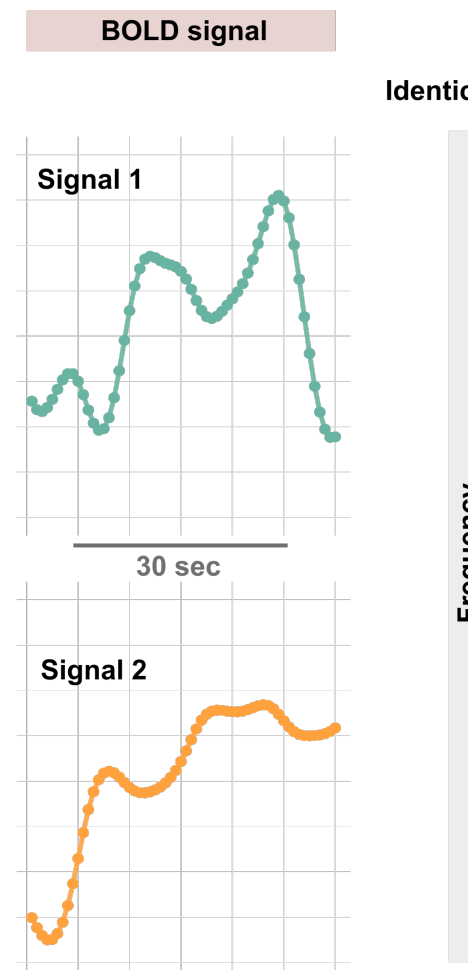

Time [TRs]

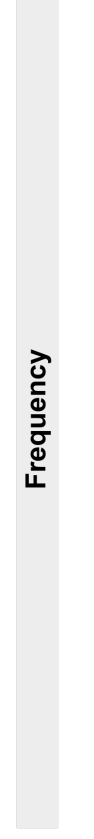

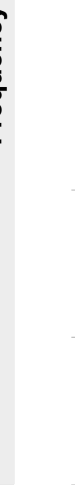

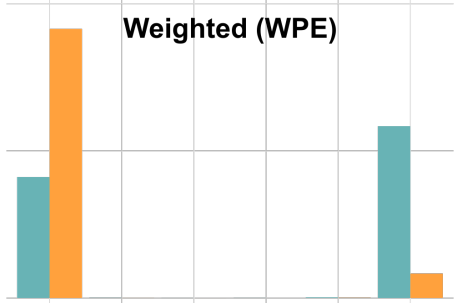

Signal 1

Signal 2

Unweighted (PE)

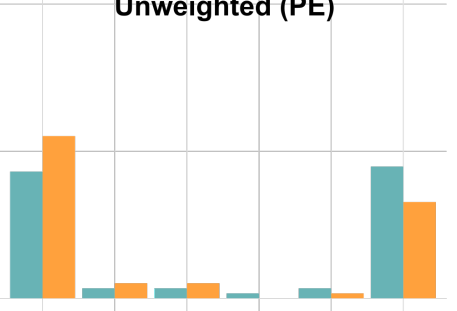

Motifs
Metric

$\triangle W P E=-0.23$

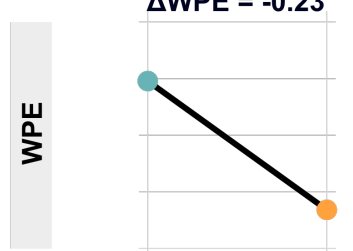

$\Delta \mathrm{PE}=\mathbf{- 0 . 0 4}$

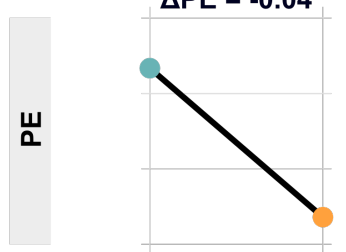

$\Delta \operatorname{Var}=\mathbf{0}$

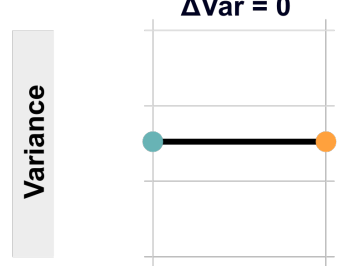

Signal 1

Signal 2

Participant ID: 102816, REST 1

b

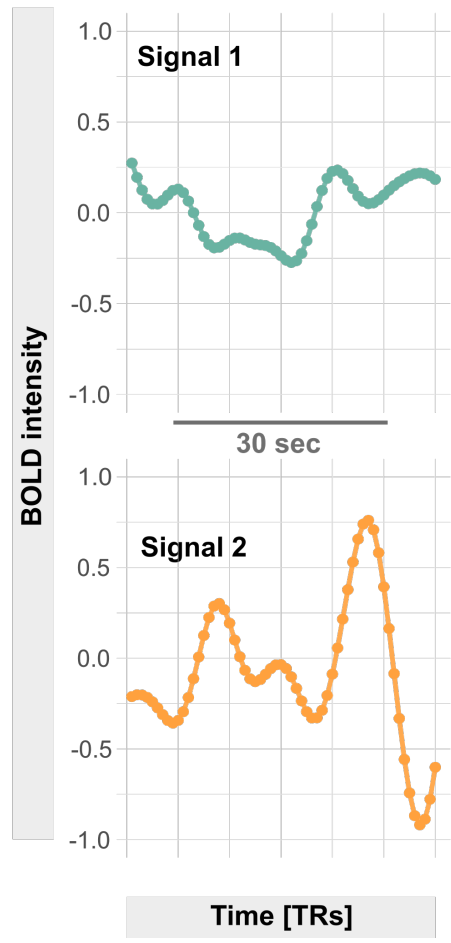

Identical pattern diversity, difference in amplitude variance

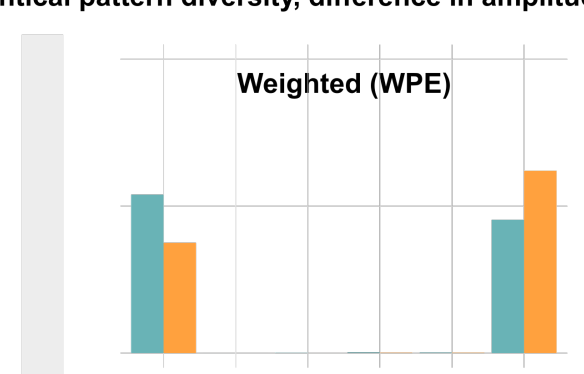

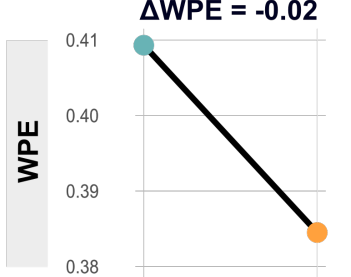

$\Delta P E=0$
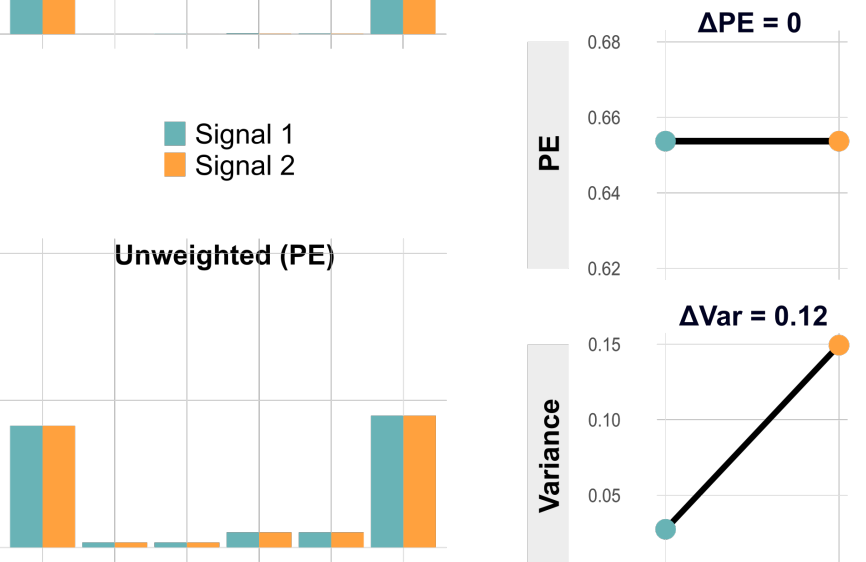

Motifs
Signal 1

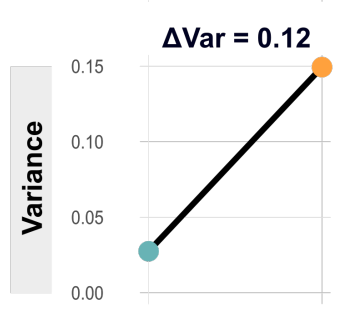

Signal 2

Extended Data Fig.2 | Weighted permutation entropy tracks BOLD signal dynamics that remain undetected when considering only pattern diversity or signal variance alone. $a, B O L D$ signals with identical amplitude variance but varying pattern diversity (permutation entropy, PE). $\mathbf{b}$, BOLD signals with identical pattern diversity but varying amplitude variance. All signal snippets correspond to non-overlapping BOLD windows (60 TRs, as in main text) from the right postcentral gyrus of a representative resting-state recording. Columns show BOLD signals (left), the associated variance-weighted pattern distributions (middle), and the change in signal metrics (right). 


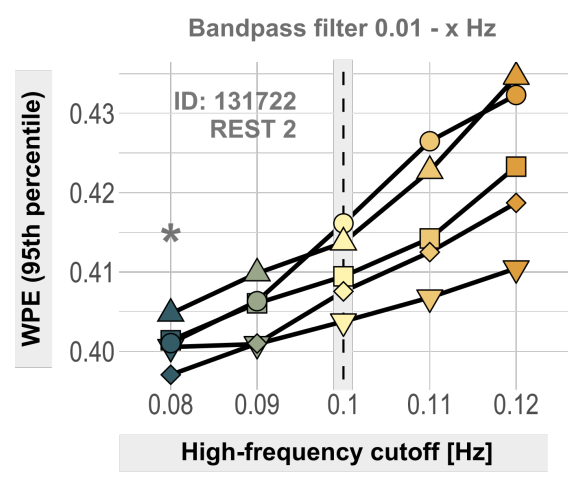

$\triangle$ right insular gyrus (174)

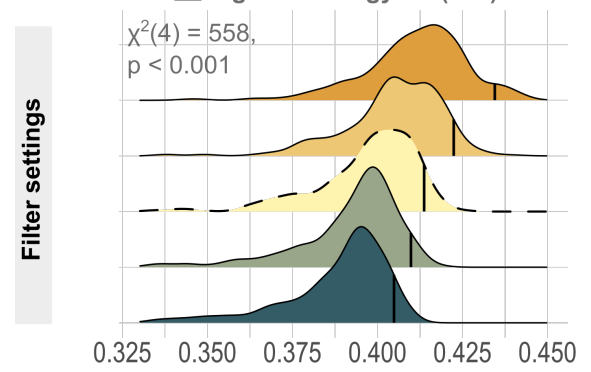

$\nabla$ right medioventral occ. cortex (190)

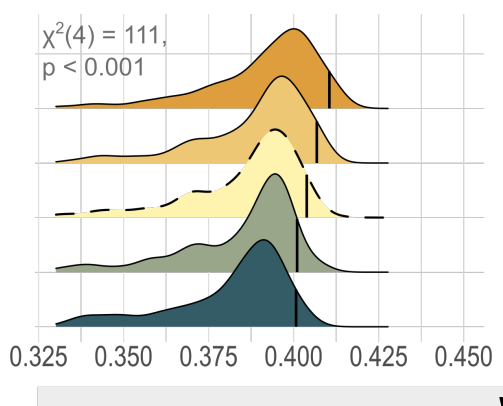

right cingulate gyrus (180)

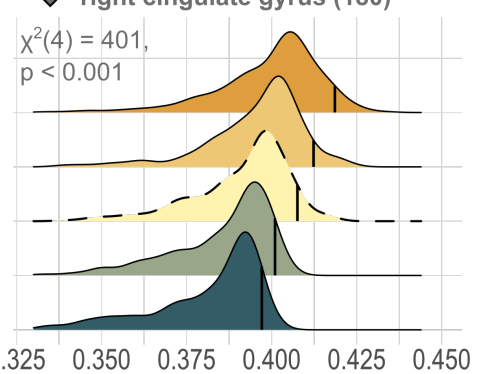

WPE left superior frontal gyrus (11)

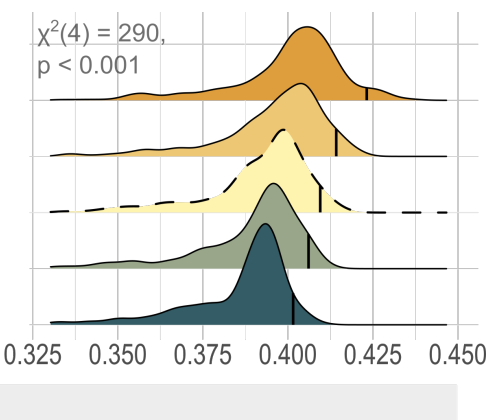

right inferior temporal gyrus (94)

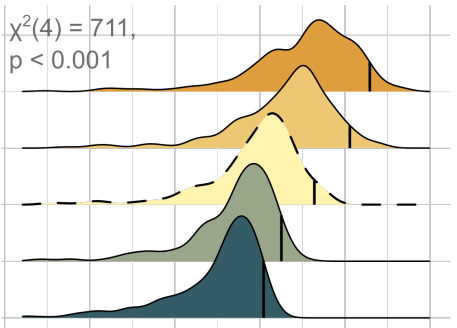

$\begin{array}{llllll}0.325 & 0.350 & 0.375 & 0.400 & 0.425 & 0.450\end{array}$

Extended Data Fig.3 | Higher frequency content scales the upper limit on weighted permutation entropy values. The upper left panel shows the $95^{\text {th }}$ percentile of WPE values from five randomly selected ROls over different bandpass filter settings in a representative resting-state dataset. Brainnetome IDs of regions are given in brackets. In line with the frequency-sensitivity in pure sine signals (Extended Data Fig.1), systematic variation of the high-frequency cutoff gradually scales the upper limits of WPE values, which becomes apparent as significant monotonic upward trend in nonparametric Mann-Kendall tests and a systematic right-shift of the time-resolved WPE distributions for individual regions. For the applied low-pass cutoff of $0.1 \mathrm{~Hz}$ in the main text (dashed lines), maximum WPE values converge around 0.42 both in simulated and real signals, in line with the empirically observed upper limit of individual complexity timeseries (Fig.1a; online repository: https://osf.io/mr8f7; Extended Data Fig.4). 


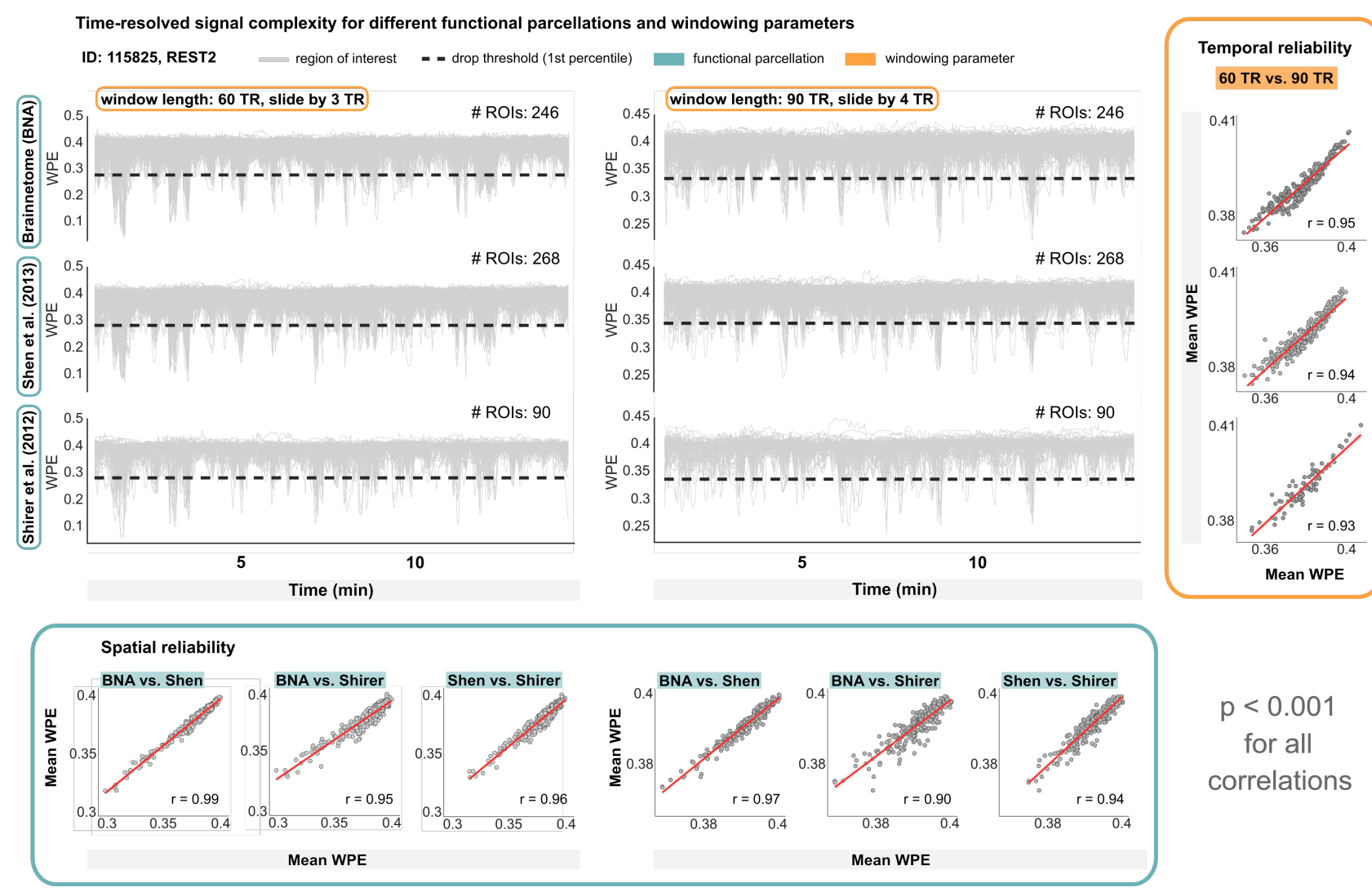

Extended Data Fig.4 | Reliability of resting-state complexity dynamics across functional parcellation granularity and windowing parameters. Time-resolved weighted permutation entropy from an exemplary HCP scan. The data were parcellated with three common functional brain atlases of varying spatial granularity (Brainnetome after Fan et al. ${ }^{59}$, Shen et al. ${ }^{71}$, and Shirer et al. ${ }^{72}$ ) and with different windowing parameters for computing the WPE (60 TRs sliding by 3 TRs, and 90 TRs sliding by 4TRs). Spatial reliability is calculated as the correlation over time points for every combination of functional atlases, and temporal reliability is computed as the correlation of mean WPE values of every ROI for the two windowing parameters. Results in the main text are based on the Brainnetome atlas and 60 TR windows sliding to the next window by 3 TRs (upper left panel here). 


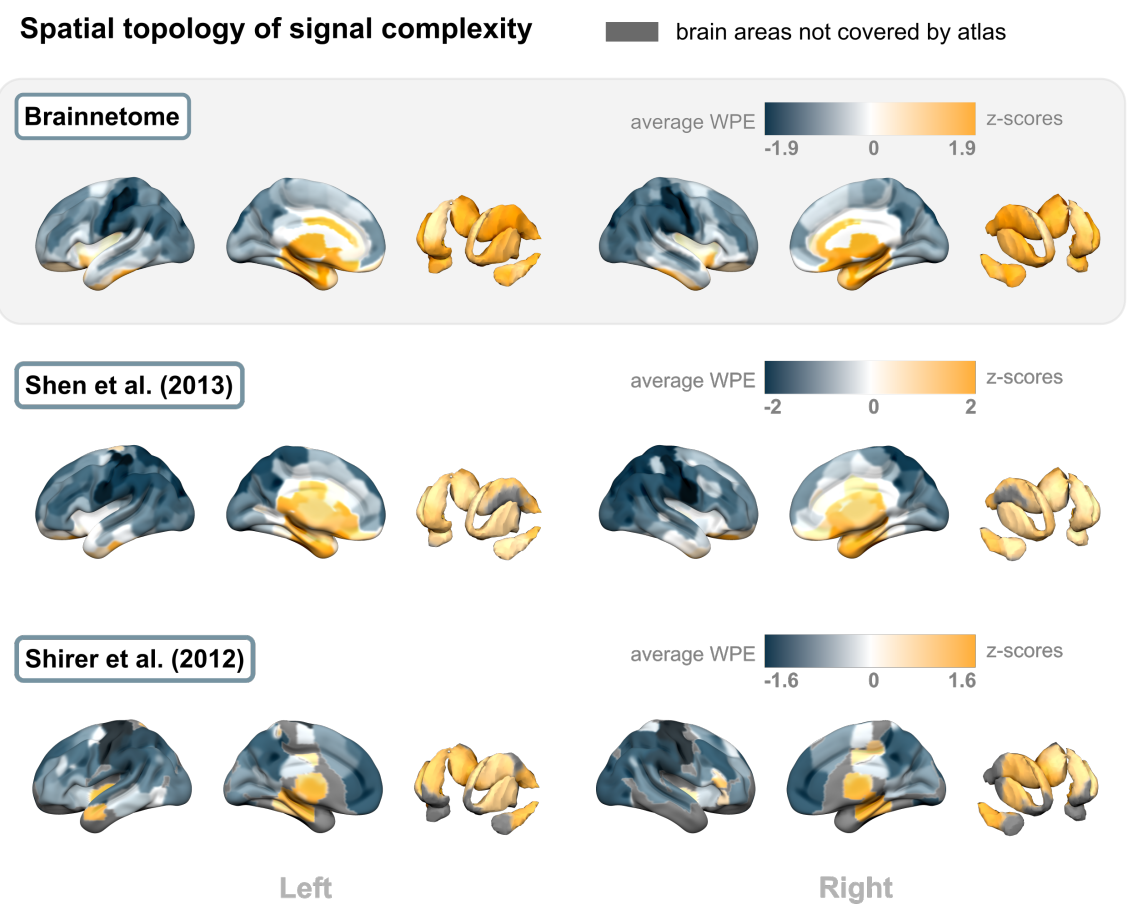

Extended Data Fig.5 | The group-level spatial topology of signal complexity is robust across functional parcellation granularities. Spatial distribution of weighted permutation entropy averaged across participants. The same three functional brain atlases as in Extended Data Fig. 3 were used (Brainnetome after Fan et al. ${ }^{59}$, Shen et al. ${ }^{71}$, and Shirer et al. ${ }^{72}$ ). The results from the main text are based on the Brainnetome atlas (gray background). The divergence between subcortical regions (high complexity) and cortex, especially in pericentral areas (low complexity), is equivalently observed with the other two atlases of higher (Shen) and lower (Shirer) spatial granularity. 
a State distributions Occupancy

$x^{2}(2)=827, p<0.001$

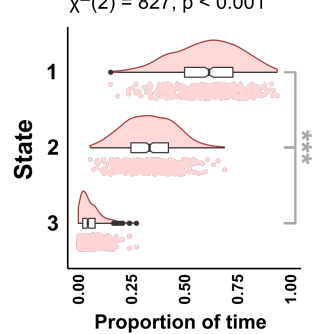

C Centroid topology over complexity states
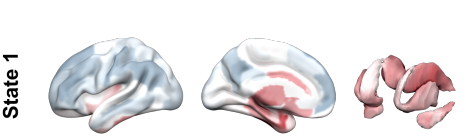

के

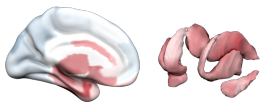

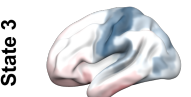

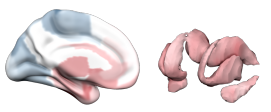

$\mathbf{k}=3$ states

Left

d

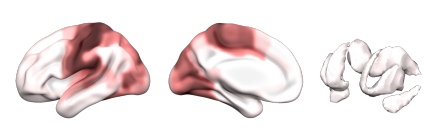

Across-state distance (ASD)

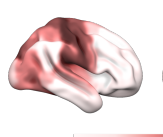

o
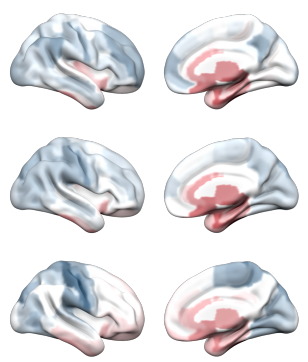

ASD vs. cortical myelin: $\varrho=0.68, p_{\text {spin }}<0.001$

ASD vs. FC gradient 1: $\varrho=-0.76, p_{\text {spin }}<0.001$
Right

edge-wise derivation

pairwise $* * * p<0.001 \quad * * p<0.01$

Modularity Efficiency

$x^{2}(2)=153, p<0.001$

$x^{2}(2)=182, p<0.001$

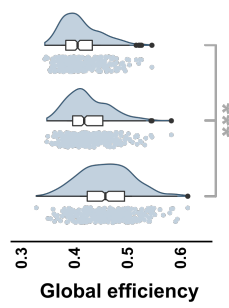

Complexity

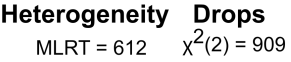
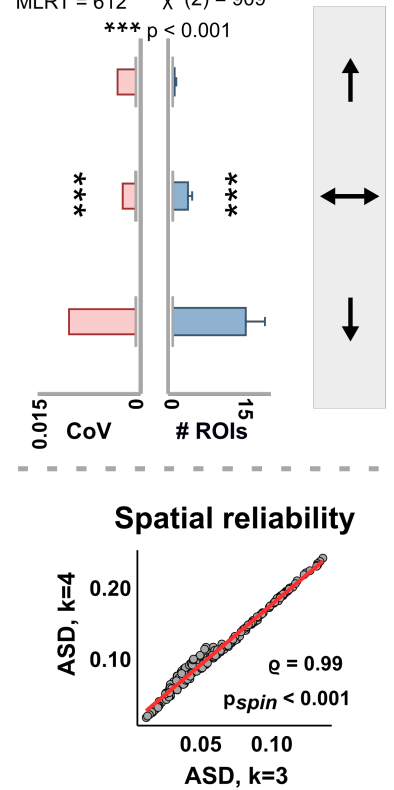

b State dispersion over $k=3$ states

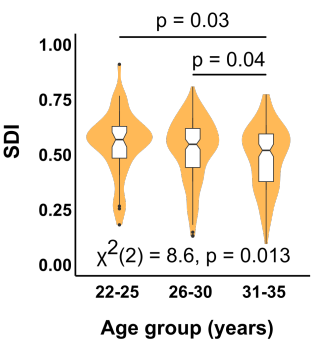

e Partial correlation (e)

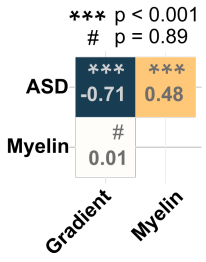

f Hierarchical regression

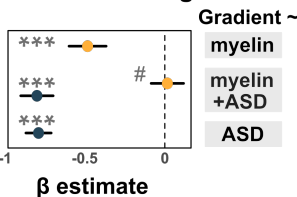

$* * * p<0.001 \quad \# p>0.05$

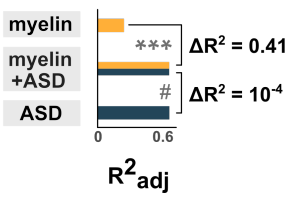

Extended Data Fig.6 | Control analyses for $\mathbf{k}=3$ expected complexity states. a, State-wise distributions of occupancy, signal complexity, FC strength, network modularity, and global efficiency. b. Age-related decline of state dispersion. c, Spatial topology, heterogeneity, and state-wise drop distribution over complexity states. d, Topology and spatial reliability of the across-state distance (ASD) compared to $k=4$ expected complexity states. e, Partial correlation between ASD, myelin, and the uni- to transmodal connectivity gradient loadings. $f$, Hierarchical regression on the gradient loadings with myelin content and ASD as explanatory variables. All results closely follow the results reported in Fig. 3 of the main text. 
a State distributions Occupancy

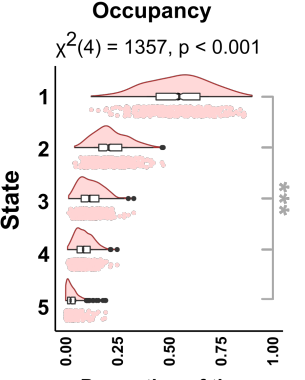

$$
\text { Proportion of time }
$$

node-wise derivation

$$
\text { Complexity }
$$$$
x^{2}(4)=1501, p<0.001
$$

edge-wise derivation

Connectivity
$x^{2}(4)=266, p<0.001$
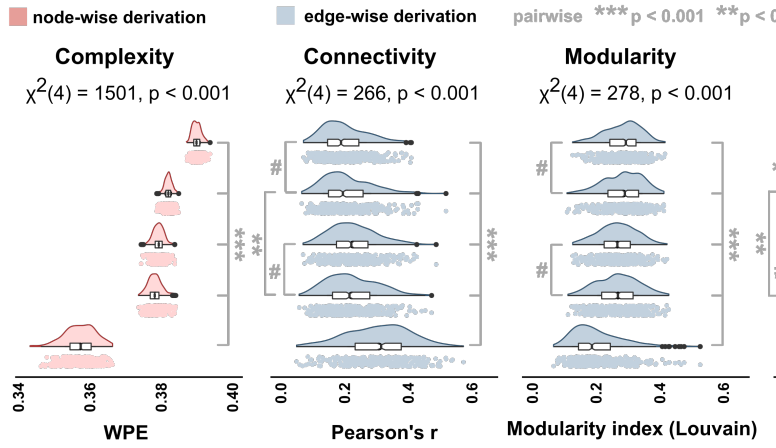

\section{1}

Heterogeneity Drops Complexity

Centroid topology over complexity states
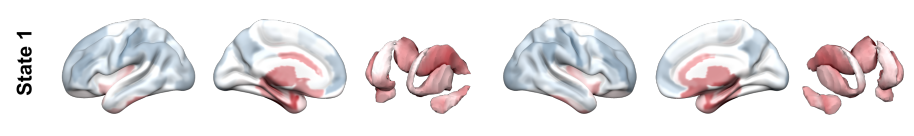

के
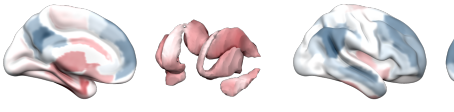

के

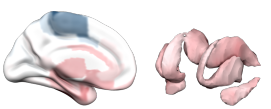

के
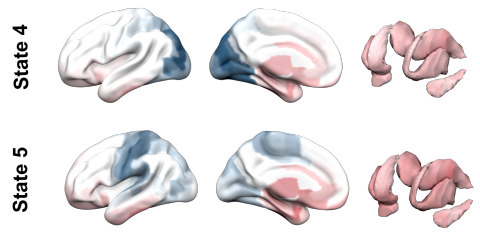

$\mathbf{k}=\mathbf{5}$ states

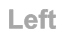

d

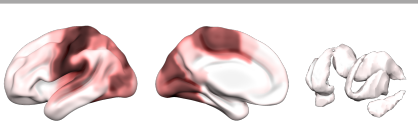

Across-state distance (ASD)

ASD vs. cortical myelin: $\varrho=0.68, p_{\text {spin }}<0.001$

ASD vs. FC gradient 1 : $\varrho=-0.76, p_{\text {spin }}<0.001$ MLRT $=914 \quad X^{2}(4)=1457$
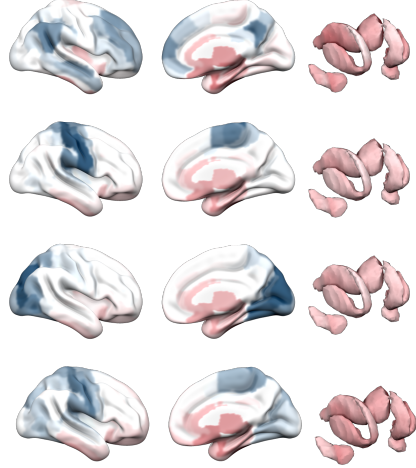

Right

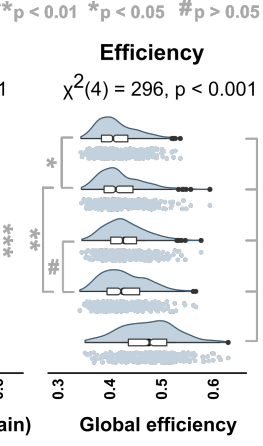

b

State dispersion over

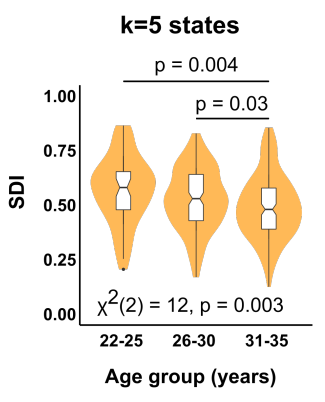

e Partial correlation (e)

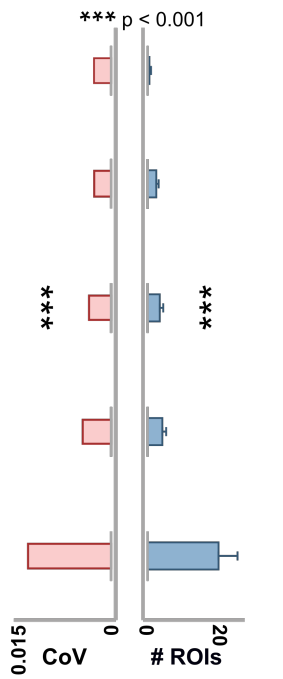

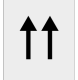
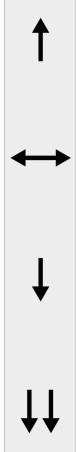

$$
\begin{aligned}
& \begin{array}{r}
* * * p<0.001 \\
\# p=0.88 \\
* * *
\end{array} \\
& \text { ASD } \begin{array}{ll}
-0.65 & 0.51
\end{array} \\
& \text { Myelin \# } \\
& \sigma^{x^{20}} e^{e^{x}}
\end{aligned}
$$

f Hierarchical regression

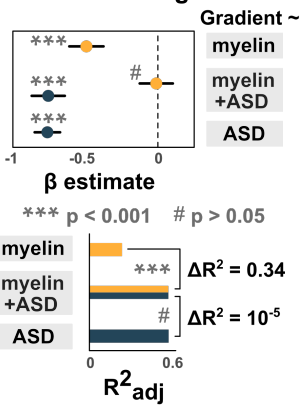

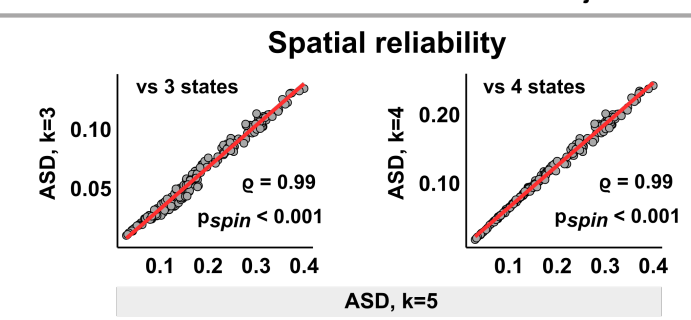

Extended Data Fig.7 | Control analyses for k=5 expected complexity states. a, State-wise distributions of occupancy, signal complexity, FC strength, network modularity, and global efficiency. b, Age-related decline of state dispersion. c, Spatial topology, heterogeneity, and state-wise drop distribution over complexity states. d, Topology and spatial reliability of the across-state distance (ASD) compared to $k=4$ and $k=3$ expected complexity states. e, Partial correlation between ASD, myelin, and the uni- to transmodal connectivity gradient loadings. $f$, Hierarchical regression on the gradient loadings with myelin content and ASD as explanatory variables. As for $k=3$ expected complexity states (Extended Data Fig.6), results closely follow the results reported in Fig. 3 of the main text. 
a

a Manhattan plot of complexity-behavior associations

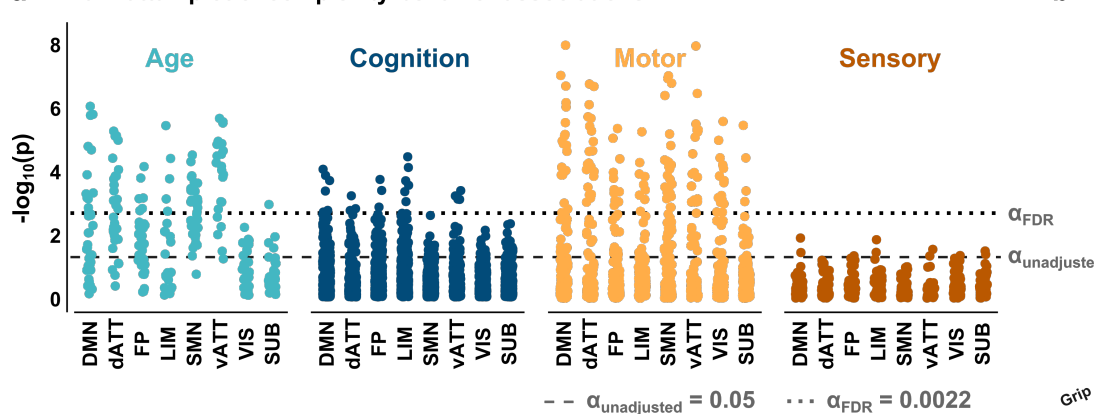

b

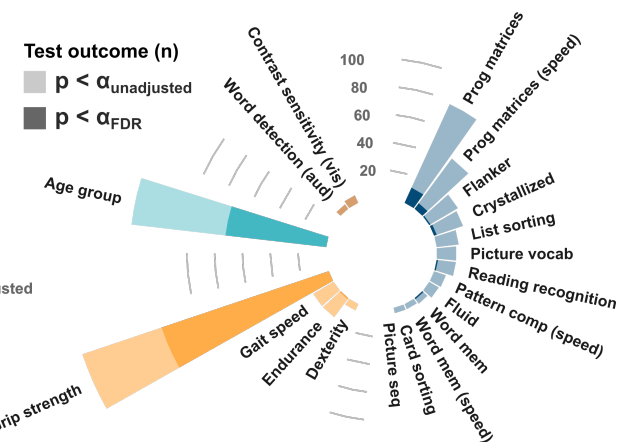

Extended Data Fig.8 | Univariate complexity-behavior associations over individual scores. a, Manhattan plot of negative log p-values for nonparametric correlation tests between behavioral variables and regional WPE values. (dashed line: unadjusted alpha-level of 0.05 ; dotted line: critical alpha-level after FDR-correction over all tests). b. Test outcomes over the individual scores of age, cognition, motor function, and sensory task performance. To extract general behavioral features, dimensionality reduction through Principal Component Analysis was applied to individual task performance metrics for the PLS analysis in the main text. 